\title{
Reflection K-Matrices for 19-Vertex Models
}

\author{
A. Lima-Santos 用 \\ Universidade Federal de São Carlos, Departamento de Física \\ Caixa Postal 676, CEP 13569-905 São Carlos, Brasil
}

\begin{abstract}
We derive and classify all regular solutions of the boundary Yang-Baxter equation for 19-vertex models known as Zamolodchikov-Fateev or $A_{1}^{(1)}$ model, Izergin-Korepin or $A_{2}^{(2)}$ model, $\operatorname{sl}(2 \mid 1)$ model and the $\operatorname{osp}(2 \mid 1)$ model. We find that there is a general solution for the $A_{1}^{(1)}$ and $s l(2 \mid 1)$ models. In both models it is a complete K-matrix with three free parameters. For the $A_{2}^{(2)}$ and $\operatorname{ssp}(2 \mid 1)$ models we find three general solutions, being two complete reflection K-matrices solutions and one incomplete reflection K-matrix solution with some null entries. In both models these solutions have two free parameters. Integrable spin-1 Hamiltonians with general boundary interactions are also presented. Several reduced solutions from these general solutions are presented in the appendices.
\end{abstract}

September 26, 2018

*e-mail: dals@power.ufscar.br 


\section{Introduction}

In the study of the two-dimensional integrable systems of quantum field theories and statistical physics the Yang-Baxter (YB) equation plays essential roles in establishing the integrability and in solving the models.

Each solution of the YB equation can be treated as a factorized $S$-matrix in some 1+1-dimensional field theory [1, 2]. In statistical physics the YB solution is treated as a vertex weight matrix of an exactly solvable statistical model on a plane lattice.

The central object in the theory of integrable models [3, 4] is the $R$-matrix $R(u)$, where $u$ is the spectral parameter. It acts on the tensor product $V^{1} \otimes V^{2}$ for a given vector space $V$ and satisfy a special system of algebraic functional equations, the YB equation

$$
R_{12}(u) R_{13}(u+v) R_{23}(v)=R_{23}(v) R_{13}(u+v) R_{12}(u)
$$

in $V^{1} \otimes V^{2} \otimes V^{3}$, where $R_{12}=R \otimes \mathbf{1}, R_{23}=\mathbf{1} \otimes R$, etc.

An $R$ matrix is said to be regular if has the property $R(0)=P$, where $P$ is the permutation matrix in $V^{1} \otimes V^{2}: P(|\alpha\rangle \otimes|\beta\rangle)=|\beta\rangle \otimes|\alpha\rangle$ for $|\alpha\rangle,|\beta\rangle \in V$. To every regular $R$-matrix there corresponds a periodic integrable quantum spin chain, with Hamiltonian $H$ given by

$$
H=\sum_{k=1}^{N-1} H_{k, k+1}+H_{N, 1},
$$

where the two-site Hamiltonian $H_{k, k+1}$ is given by

$$
H_{k, k+1}=\left.P \frac{d}{d u} R(u)\right|_{u=0} .
$$

The right hand side of (1.3) is acting on the quantum spaces at sites $k$ and $k+1$.

An $R$-matrix is said to be quasi-classical if it depends on an additional parameter $\eta$ (playing the role of the Planck constant), so that for small $\eta$

$$
R(u, \eta)=1+2 \eta r(u)+O\left(\eta^{2}\right)
$$

The quantity $r(u)$ is called a classical $r$-matrix.

Many regular quasi-classical solutions are related to the simple Lie (super) algebra [5, 6, 7, 8]. They are classified by irreducible representations of the Lie algebra in elliptic, trigonometric and rational, corresponding to the three types of function of the spectral parameter $u$.

Recently there has been a lot of efforts in introducing boundaries into integrable systems for possible applications to condensed matter physics and statistical system 
with non-periodic boundary conditions. The boundaries entail new physical quantities called reflection matrices which depend on the boundary properties.

By considering systems on a finite interval with independent boundary conditions at each end, we have to introduce reflection matrices to describe such boundary conditions. Integrable models with boundaries can be constructed out of a pair of reflection $K$ matrices $K_{ \pm}(u)$ in addition to the YB $R$-matrix. $K_{+}(u)$ and $K_{-}(u)$ describe the effects of the presence of boundaries at the left and the right ends, respectively.

Integrability of open chains in the framework of the quantum inverse scattering method was pioneered by Sklyanin relying on previous results of Cherednik [9]. In reference [10], Sklyanin has used his formalism to solve, via algebraic Bethe ansatz, the open spin-1/2 chain with diagonal boundary terms. This model had already been solved via coordinate Bethe ansatz by Alcaraz et al [11.

The Sklyanin original formalism was extended to more general systems by Mezincescu and Nepomechie in [12], where is assumed that for a regular $R$ matrix satisfying the following properties

$$
\begin{aligned}
& \text { PT-symmetry : } P_{12} R_{12}(u) P_{12}=R_{21}(u), \\
& \text { unitarity : } R_{12}(u) R_{21}(-u) \propto 1 \text {, } \\
& \text { crossing unitarity }: R_{12}(u)=(U \otimes 1) R_{12}^{\mathrm{t}_{2}}(-u-\rho)(U \otimes 1)^{-1} \text {, }
\end{aligned}
$$

one can derive an integrable open chain Hamiltonian

$$
H=\sum_{k=1}^{N-1} H_{k, k+1}+\frac{1}{2}\left(\left.\frac{d K_{-}(u)}{d u}\right|_{u=0} \otimes 1\right)+\frac{\operatorname{tr}_{0} \stackrel{0}{K}_{+}(0) H_{N, 0}}{\operatorname{tr} K_{+}(0)}
$$

where $H_{k, k+1}$ is given by $(1.3)$ and $K_{-}(u)$ is the reflection matrix which satisfy the right boundary YB equation, also known as the right reflection equation (RE)

$$
\begin{gathered}
R_{12}(u-v)\left(K_{-}(u) \otimes 1\right) R_{21}(u+v)\left(1 \otimes K_{-}(v)\right)= \\
\left(1 \otimes K_{-}(v)\right) R_{12}(u+v)\left(K_{-}(u) \otimes 1\right) R_{21}(u-v)
\end{gathered}
$$

and $K_{+}(u)$ is the reflection matrix which satisfy an left RE.

Given a solution $K_{-}(u)$ of $(1.7)$, one can show that the corresponding quantity

$$
K_{+}(u)=K_{-}^{\mathrm{t}}(-u-\rho) M, \quad M=U^{\mathrm{t}} U=M^{\mathrm{t}}
$$

satisfy the left RE. Here $\rho$ is a crossing parameter and $U$ is a crossing matrix both being specific to each model [6, 8]. $\mathrm{t}_{i}$ stands for the transposition taken in the $i$-th space and $\operatorname{tr}_{0}$ is the trace taken in the auxiliary space. 
Although a careful analysis within the framework of algebraic structures was carried out in [13, 14, 15], unlike $R$-matrix solutions there is no direct relation of the quasiclassical $K$-matrix solutions to the Lie (super) algebra theory. In particular, many of them do not depend on the quasiclassical parameter $\eta$.

In field theory, attention is focused on the boundary $S$-matrix and recent field theoretical applications of the RE can be found in [16, 17, 18, 19]. In statistical mechanics, the emphasis has been on deriving solutions of the $\mathrm{RE}$ and the investigation of various surface critical phenomena, both at and away from criticality [20]. In condensed matter physics, the Kondo problem with integrable boundary impurities has been studied by means of the boundary graded quantum inverse scattering method [21].

Due to the significance of the RE, a lot of work has been directed to the study of their solutions [9, 22, 23, 24]. There is a classification of reflection solutions for twocomponent systems [25]. Batchelor at al [26] have derived diagonal solutions of the RE for the face and vertex models related to affine Lie algebras. Complete nondiagonal reflection matrices of face-type models as SOS, RSOS and the hard hexagonal model were recently derived by Ahn and You [27]. Diagonal solutions for fused high spin models were presented by Abad and Rios [28].

In this paper we consider the 19-vertex models known as Zamolodchikov-Fateev or $A_{1}^{(1)}$ model, Izergin-Korepin or $A_{2}^{(2)}$ model, the $s l(2 \mid 1)$ model and the $o s p(2 \mid 1)$ model. These vertex models have a common algebraic structure which permits us to solve their RE in an unified way. General regular solutions are derived and classified into two categories: Type-I solution, which is the complete reflection $K$-matrix of each 19vertex model, and Type-II solution, which is an additional solution for the IK model and the $\operatorname{ssp}(2 \mid 1)$ model. These solutions were computed in a direct way by solving functional equations.

The paper is organized as follows. In section 2 we present a procedure to derive general solutions of the RE for these models. In section 3 we carry out the procedure explicitly for the ZF model. Here one can see that our result is in agreement with the previous result derived by Inami et al [24]. In section 4 the type-I and type-II general solutions are calculated for the IK model. In sections 5 and 6 we present the regular reflection $K$-matrices for the graded $s l(2 \mid 1)$ and $\operatorname{ssp}(2 \mid 1)$ models, respectively. In section 7 the corresponding integrable Hamiltonians with general boundary interactions are also presented. Our conclusions are presented in the last section. Finally, we reserved four appendices to give a sub-classification for the reduced solutions which are derived from the general ones as special limits. 


\section{General Solutions}

Along this paper we will work with both graded and non-graded models. Therefore we need to recall some informations about the graded formulation.

Let $V=V_{0} \oplus V_{1}$ be a $Z_{2}$-graded vector space where 0 and 1 denote the even and odd parts respectively. Multiplication rules in the graded tensor product space $V \stackrel{s}{\otimes} V$ differ from the ordinary ones by the appearance of additional signs. The components of a linear operator $A \stackrel{s}{\otimes} B \in V \stackrel{s}{\otimes} V$ result in matrix elements of the form

$$
(A \stackrel{s}{\otimes} B)_{\alpha \beta}^{\gamma \delta}=(-)^{p(\beta)(p(\alpha)+p(\gamma))} A_{\alpha \gamma} B_{\beta \delta} .
$$

The action of the graded permutation operator $P^{\mathrm{g}}$ on the vector $|\alpha\rangle \stackrel{s}{\otimes}|\beta\rangle \in V \stackrel{s}{\otimes} V$ is defined by

$$
P^{\mathrm{g}}|\alpha\rangle \stackrel{s}{\otimes}|\beta\rangle=(-)^{p(\alpha) p(\beta)}|\beta\rangle \stackrel{s}{\otimes}|\alpha\rangle \Longrightarrow\left(P^{\mathrm{g}}\right)_{\alpha \beta}^{\gamma \delta}=(-)^{p(\alpha) p(\beta)} \delta_{\alpha \delta} \delta_{\beta \gamma} .
$$

The super-transposition st and the super-trace str are defined by

$$
\left(A^{\mathrm{st}}\right)_{\alpha \beta}=(-)^{(p(\alpha)+p(\beta)) p(\alpha)} A_{\beta \alpha}, \quad \operatorname{str} A=\sum_{\alpha}(-)^{p(\alpha)} A_{\alpha \alpha} .
$$

where $p(\alpha)=1(0)$ if $|\alpha\rangle$ is an odd (even) element.

Taking into account this new formulation, the equations (1.1) and (1.7) are now named graded YB equation and boundary graded YB equation, respectively.

The YB solution for the models which we are going to treat in this paper has a common form given by the following $R$-matrix

$$
R(u)=\left(\begin{array}{lllllllll}
x_{1} & 0 & 0 & 0 & 0 & 0 & 0 & 0 & 0 \\
0 & x_{2} & 0 & x_{5} & 0 & 0 & 0 & 0 & 0 \\
0 & 0 & x_{3} & 0 & x_{6} & 0 & x_{7} & 0 & 0 \\
0 & y_{5} & 0 & x_{2} & 0 & 0 & 0 & 0 & 0 \\
0 & 0 & y_{6} & 0 & x_{4} & 0 & x_{6} & 0 & 0 \\
0 & 0 & 0 & 0 & 0 & x_{2} & 0 & x_{5} & 0 \\
0 & 0 & y_{7} & 0 & y_{6} & 0 & x_{3} & 0 & 0 \\
0 & 0 & 0 & 0 & 0 & y_{5} & 0 & x_{2} & 0 \\
0 & 0 & 0 & 0 & 0 & 0 & 0 & 0 & x_{1}
\end{array}\right),
$$

where the non-zero entries for each model will be presented in the next sections. In the graded cases we choose a common parity assignments: $p(1)=p(3)=0, p(2)=1$.

For regular solutions $K_{-}(u)$ of the right RE we can choose the following normalization

$$
K_{-}(u)=\left(\begin{array}{ccc}
k_{11}(u) & k_{12}(u) & k_{13}(u) \\
k_{21}(u) & 1 & k_{23}(u) \\
k_{31}(u) & k_{32}(u) & k_{33}(u)
\end{array}\right)
$$


with

$$
k_{i j}(0)=0 \quad \text { for } \quad i \neq j \quad \text { and } \quad k_{i i}(0)=1, \quad i, j=1,2,3
$$

Substituting (2.4) and (2.5) into (1.7) and into its graded version, we have in both cases 81 functional equations for the $k_{i j}$ elements, many of which are dependent. In order to solve them, we shall proceed in the following way. First we consider the $(i, j)$ component of the matrix equation (1.7). By differentiating it with respect to $v$ and taking $v=0$, we will get algebraic equations involving the single variable $u$ and eight parameters

$$
\beta_{i j}=\left.\frac{d k_{i j}(v)}{d v}\right|_{v=0} \quad i, j=1,2,3 . \quad\left(\beta_{22}=0\right)
$$

Second, these algebraic equations are denoted by $E[i, j]=0$ and collected into 25 blocks $B[i, j]$ defined by

$$
\begin{aligned}
B[i, j] & =\{E[i, j]=0, E[j, i]=0, E[10-i, 10-j]=0, E[10-j, 10-i]=0\} \\
i & =1, \ldots, 5 \quad \text { and } \quad j=i, \ldots, 10-i .
\end{aligned}
$$

For a given block $B[i, j]$, the equation $E[10-j, 10-i]=0$ can be obtained from the equation $E[i, j]=0$ by interchanging

$$
\begin{aligned}
k_{i j} & \longleftrightarrow k_{4-j, 4-i}, \quad \beta_{i j} \longleftrightarrow \beta_{4-j, 4-i}, \quad x_{i} \longleftrightarrow y_{i} \quad \text { for } \quad i=5,7 \\
\text { and } \quad x_{6} & \longleftrightarrow y_{6}
\end{aligned}
$$

and the equation $E[j, i]=0$ is obtained from the equation $E[i, j]=0$ by the interchanging

$$
k_{i j} \longleftrightarrow k_{j i}, \quad \beta_{i j} \longleftrightarrow \beta_{j i}, \quad x_{6} \longleftrightarrow \epsilon x_{6}, \quad y_{6} \longleftrightarrow \epsilon y_{6},
$$

where $\epsilon$ makes the difference among graded and non-graded models: $\epsilon=1$ for the ZF and IK models and $\epsilon=-1$ for the $\operatorname{sl}(2 \mid 1)$ and $\operatorname{osp}(2 \mid 1)$ models.

In order to give an example, let us consider the block $B[2,8]$ where we have the two simplest equations:

$$
\begin{aligned}
& E[2,8]=\left[\beta_{12} x_{2} y_{6}-\beta_{23} x_{3} y_{5}\right] k_{12}-\left[\epsilon \beta_{23} x_{2} x_{6}-\beta_{12} x_{3} x_{5}\right] k_{23}=0 \\
& E[8,2]=\left[\epsilon \beta_{21} x_{2} y_{6}-\beta_{32} x_{3} y_{5}\right] k_{21}-\left[\beta_{32} x_{2} x_{6}-\beta_{21} x_{3} x_{5}\right] k_{32}=0 .
\end{aligned}
$$

Note that we can use (2.10) to write the equation $E[8,2]=0$ from the equation $E[2,8]=0$. Therefore, using the interchanging rules (2.9) and (2.10) for each block $B[i, j]$ we only needed to look at the equation $E[i, j]=0$, which will itself be identified with the block 
Combining the two equations of the block $B[2,8]$ with the four equations of the block

$$
B[1,6]=\beta_{13} x_{2}\left(x_{1}-x_{3}\right) k_{12}-\epsilon \beta_{13} x_{5} x_{6} k_{23}-\left[\beta_{12} x_{2} x_{5}-\epsilon \beta_{23} x_{1} x_{6}\right] k_{13},
$$

and with the four equations of the block

$$
B[1,8]=\left[x_{2}^{2}-x_{1} x_{3}\right] \beta_{12} k_{13}+\beta_{13} x_{3} y_{5} k_{12}+\epsilon \beta_{13} x_{2} x_{6} k_{23},
$$

we will get the relations

$$
\begin{array}{ll}
k_{12}=\frac{\beta_{12} x_{3} x_{5}-\epsilon \beta_{23} x_{2} x_{6}}{x_{3}\left(x_{1}-x_{3}\right)+\epsilon x_{6} y_{6}} \frac{k_{13}}{\beta_{13}}, \quad k_{23}=\frac{\beta_{23} x_{3} y_{5}-\beta_{12} x_{2} y_{6}}{x_{3}\left(x_{1}-x_{3}\right)+\epsilon x_{6} y_{6}} \frac{k_{13}}{\beta_{13}}, \\
k_{21}=\frac{\beta_{21} x_{3} x_{5}-\beta_{32} x_{2} x_{6}}{x_{3}\left(x_{1}-x_{3}\right)+\epsilon x_{6} y_{6}} \frac{k_{31}}{\beta_{31}}, \quad k_{32}=\frac{\beta_{32} x_{3} y_{5}-\epsilon \beta_{21} x_{2} y_{6}}{x_{3}\left(x_{1}-x_{3}\right)+\epsilon x_{6} y_{6}} \frac{k_{31}}{\beta_{31}} .
\end{array}
$$

Here we have used the following identity

$$
x_{3}\left(x_{1}^{2}+x_{2}^{2}\right)+\epsilon x_{1} x_{6} y_{6}=x_{1}\left(x_{2}^{2}+x_{3}^{2}\right)+x_{3} x_{5} y_{5}
$$

which is holds for all above-mentioned 19-vertex models.

Next we consider the nine equations of the blocks $B[i, i]$

$$
\begin{aligned}
B[1,1]= & x_{1} x_{5}\left(\beta_{21} k_{12}-\beta_{12} k_{21}\right)+x_{1} x_{7}\left(\beta_{31} k_{13}-\beta_{13} k_{31}\right), \\
B[2,2]= & x_{1} x_{5}\left(\beta_{12} k_{21}-\beta_{21} k_{12}\right)+x_{5} x_{7}\left(\beta_{32} k_{23}-\beta_{23} k_{32}\right) \\
& +x_{2} x_{6}\left(\beta_{32} k_{12}-\epsilon \beta_{23} k_{21}\right), \\
B[3,3]= & x_{1} x_{7}\left(\beta_{13} k_{31}-\beta_{31} k_{13}\right)-x_{5} x_{7}\left(\beta_{32} k_{23}-\beta_{23} k_{32}\right) \\
& +x_{2} x_{6}\left(\epsilon \beta_{23} k_{21}-\beta_{32} k_{12}\right), \\
B[4,4]= & \epsilon x_{4} y_{5}\left(\beta_{21} k_{12}-\beta_{12} k_{21}\right)-x_{5} y_{5}\left(\beta_{13} k_{31}-\beta_{31} k_{13}\right) \\
& +x_{2} x_{6}\left(\epsilon \beta_{21} k_{23}-\beta_{12} k_{32}\right), \\
B[5,5]= & -\epsilon x_{4} y_{5}\left(\beta_{21} k_{12}-\beta_{12} k_{21}\right)+\epsilon x_{4} x_{5}\left(\beta_{32} k_{23}-\beta_{23} k_{32}\right) \\
& -x_{2} x_{6}\left(\epsilon \beta_{23} k_{21}-\beta_{32} k_{12}\right)+x_{2} y_{6}\left(\beta_{32} k_{12}-\epsilon \beta_{23} k_{21}\right),
\end{aligned}
$$

By direct inspection one can see that these equations are solved by the relations

$$
\beta_{i j} k_{j i}(u)=\beta_{j i} k_{i j}(u) \quad i \neq j
$$

provided that

$$
\beta_{12} \beta_{32}=\epsilon \beta_{21} \beta_{23}
$$


Substituting (2.18) and (2.19) into the remaining blocks we verify that the equations $E[i, j]=0$ and $E[j, i]=0$ give the same result provided that

$$
\beta_{13} \beta_{21}^{2}=\epsilon \beta_{31} \beta_{12}^{2}
$$

Equation (2.18) is invariant under the interchange rules (2.9) and (2.10) while equation (2.20) has an interchanged form involving $\beta_{23}$ and $\beta_{32}$.

At this stage we still have to solve the equations of 16 blocks $B[i, j]$ involving three functions $k_{13}(u), k_{11}(u)$ and $k_{33}(u)$ and the six parameters $\beta_{12}, \beta_{13}, \beta_{12}, \beta_{23}, \beta_{11}$ and $\beta_{33}$.

Next, from each equation of the blocks $B[i, j]$ we express $k_{13}(u)$ in terms of $k_{11}(u)$ and $k_{33}(u)$. Equating these results we will get constraint equations for the parameters $\beta_{i j}$. This procedure will leave us with many constraint equations. For instance, from the blocks $B[1,4]$ and $B[2,6]$ we compare the results for $k_{13}(u)$ to get the following equation:

$$
\begin{gathered}
\epsilon \beta_{12} \beta_{21} \beta_{13}^{2} x_{1} x_{6}-\epsilon \beta_{21} \beta_{13}^{2}\left[\Gamma_{1} x_{6} y_{5}+\Gamma_{2} x_{2} x_{7}\right]-2 \beta_{12} \beta_{13} \Gamma_{1} w\left(x_{1}, x_{2}\right) \\
+\beta_{12}^{2} \beta_{23} \Gamma_{1} x_{2}\left(\epsilon x_{4}-x_{3}\right)+\beta_{12}^{2} \beta_{13}\left[\beta_{33} x_{2} x_{5}-2 w\left(x_{2}, x_{5}\right)\right]=0
\end{gathered}
$$

Using the interchange rule (2.9) and the relations (2.19) and (2.20) we get from (2.21) another constraint equation

$$
\begin{aligned}
& \epsilon \beta_{21} \beta_{23} \beta_{13}^{2} x_{1} y_{6}-\beta_{21} \beta_{13}^{2}\left[\Gamma_{1} x_{2} y_{7}+\epsilon \Gamma_{2} x_{5} y_{6}\right]-2 \beta_{12} \beta_{13} \Gamma_{2} w\left(x_{1}, x_{2}\right) \\
& +\beta_{12}^{2} \beta_{23} \Gamma_{2} x_{2}\left(\epsilon x_{4}-x_{3}\right)+\beta_{12} \beta_{13} \beta_{23}\left[\beta_{11} x_{2} y_{5}-2 w\left(x_{2}, y_{5}\right)\right]=0
\end{aligned}
$$

Equations (2.21) and (2.22) are enough for our purpose. They involve the six remaining parameters $\beta_{i j}$ and must be valid for all values of $u$. Their solutions are model dependent and solve all other constraint equations.

The procedure to solve these equations is the following: First we write (2.21) in a factored form $F_{1}(u, \eta) F_{2}(u, \eta)=0$ such that $F_{1}(u=0, \eta)=0$ and $F_{2}(u=0, \eta) \neq 0$. Imposing that $F_{2}(0, \eta)=0$ we can find, for instance, $\beta_{21}$ in terms of $\beta_{12}, \beta_{13}, \beta_{23}$ and $\beta_{33}$. Substituting the value obtained for $\beta_{21}$ into $F_{2}(u, \eta)$ we have two possibilities. When is $F_{2}(u, \eta)=0$ it is a solution for (2.21) with four free parameters. But if $F_{2}(u, \eta) \neq 0$ it must be rewrite in another factored form $F_{3}(u, \eta) F_{4}(u, \eta)=0$ such that $F_{3}(u=0, \eta)=0$ and $F_{4}(u=0, \eta) \neq 0$. The condition $F_{4}(0, \eta)=0$ can be used to find, for instance, the value of $\beta_{33}$ in terms of $\beta_{12}, \beta_{13}$ and $\beta_{23}$. Substituting $\beta_{33}$ into $F_{4}(u, \eta)$ we proceed as before and so on until solving (2.21) for any value of $u$.

For the ZF and the $\operatorname{sl}(2 \mid 1)$ models we will get their parameters at the second step of this procedure. It means that we have a general solution with three free parameters. 
However, for the IK and $\operatorname{osp}(2 \mid 1)$ models we will need an additional step that will supply us with the following equation

$$
F_{5}(u, \eta)\left[\beta_{12}^{2}+\epsilon \mathrm{e}^{4 \epsilon \eta} \beta_{23}^{2}\right]=0
$$

and its interchanged form

$$
G_{5}(u, \eta)\left[\beta_{21}^{2}+\epsilon \mathrm{e}^{4 \epsilon \eta} \beta_{32}^{2}\right]=0
$$

Here we observe that the equations $\beta_{12}^{2}+\epsilon \mathrm{e}^{4 \epsilon \eta} \beta_{23}^{2}=0$ and $\beta_{21}^{2}+\epsilon \mathrm{e}^{4 \epsilon \eta} \beta_{32}^{2}=0$ will give us two general solutions with two free parameters. Nevertheless, a third solution with $\beta_{12}=\beta_{21}=\beta_{23}=\beta_{32}=0$ must also be considered. It is, by construction, a new solution which also has two free parameters. In that way, we will have two types of solutions: Type-I defined when all parameters are non-vanishing and Type-II defined when the parameters $\beta_{12}, \beta_{21}, \beta_{23}$ and $\beta_{32}$ vanish.

\subsection{Type-I Solution}

Taking into account the values of the parameters $\beta_{i j} \neq 0$ which satisfy the constraint equations (2.21) and (2.22) we can choose the blocks $B[1,4]$ and $B[2,4]$ to write expressions for $k_{13}, k_{11}$ and $k_{33}$. Moreover, choosing $\beta_{32}$ and $\beta_{31}$ as the parameters fixed by the relations (2.19) and (2.20) respectively, we can also recall the relations (2.14) and (2.15) to write the type-I solution. It is a complete reflection $K$-matrix with the following elements:

$$
\begin{aligned}
& k_{12}(u)=\frac{1}{\beta_{13}} \Gamma_{1}(u) k_{13}(u), \quad k_{21}(u)=\frac{\beta_{21}}{\beta_{12} \beta_{13}} \Gamma_{1}(u) k_{13}(u), \\
& k_{23}(u)=\frac{1}{\beta_{13}} \Gamma_{2}(u) k_{13}(u), \quad k_{32}(u)=\frac{\epsilon \beta_{21}}{\beta_{12} \beta_{13}} \Gamma_{2}(u) k_{13}(u), \\
& k_{31}(u)=\frac{\epsilon \beta_{21}^{2}}{\beta_{12}^{2}} k_{13}(u), \\
& k_{13}(u)=\frac{1}{\mathcal{D}}\left[-2 \beta_{12}^{2} \beta_{13} x_{2}\left(x_{5} w\left(x_{2}, y_{5}\right)+y_{5} w\left(x_{2}, x_{5}\right)\right)\right] \\
& k_{11}(u)=\frac{1}{\mathcal{D}}\left\{( 2 w ( x _ { 2 } , x _ { 5 } ) + \beta _ { 1 1 } x _ { 2 } x _ { 5 } ) \left[\beta_{13} \beta_{21}\left(\Gamma_{1} x_{6} y_{5}+\Gamma_{2} x_{2} x_{7}\right)\right.\right. \\
& \left.-\epsilon \beta_{12} \beta_{13} \beta_{21} x_{1} x_{6}+2 \beta_{12} \Gamma_{1} w\left(x_{1}, x_{2}\right)\right]-\beta_{21} x_{2} x_{5}\left[\epsilon \beta_{13}^{2} \beta_{21} x_{2} x_{5}\right. \\
& \left.\left.+\beta_{12}^{2}\left(\epsilon \Gamma_{1} x_{2} x_{4}-\Gamma_{1} x_{1} x_{2}+\epsilon \Gamma_{2} x_{5} x_{6}\right)-\epsilon \beta_{12} \beta_{23}\left(\Gamma_{1} x_{6} y_{5}+\Gamma_{2} x_{2} x_{7}\right)\right]\right\} .
\end{aligned}
$$


where

$$
\Gamma_{1}(u)=\frac{\beta_{12} x_{3} x_{5}-\epsilon \beta_{23} x_{2} x_{6}}{x_{1} x_{3}-x_{3}^{2}+\epsilon x_{6} y_{6}} \quad \text { and } \quad \Gamma_{2}(u)=\frac{\beta_{23} x_{3} y_{5}-\beta_{12} x_{2} y_{6}}{x_{1} x_{3}-x_{3}^{2}+\epsilon x_{6} y_{6}}
$$

and

$$
\begin{aligned}
\mathcal{D}= & \left(2 w\left(x_{2}, y_{5}\right)-\beta_{11} x_{2} y_{5}\right)\left\{\epsilon \beta_{13} \beta_{21}\left[\Gamma_{1} x_{6} y_{5}+\Gamma_{2} x_{2} x_{7}\right]+\beta_{12}\left[2 \Gamma_{1} w\left(x_{1}, x_{2}\right)\right.\right. \\
& \left.\left.-\epsilon \beta_{13} \beta_{21} x_{1} x_{6}\right]\right\}+\epsilon \beta_{21} x_{2} y_{5}\left\{\beta_{13}^{2} \beta_{21} x_{2} x_{5}-\beta_{12} \beta_{23}\left[\Gamma_{1} x_{6} y_{5}+\Gamma_{2} x_{2} x_{7}\right]\right. \\
& \left.+\beta_{12}^{2}\left[\Gamma_{1} x_{2} x_{4}-\epsilon \Gamma_{2} x_{1} x_{2}+\Gamma_{2} x_{5} x_{6}\right]\right\} .
\end{aligned}
$$

Here we have used an additional identity

$$
\left(x_{2}^{2}-x_{1}^{2}\right) w\left(x_{1}, x_{2}\right)=x_{1} x_{5} w\left(x_{2}, y_{5}\right)+x_{2} y_{5} w\left(x_{1}, y_{5}\right)
$$

where $w(f, g)$ is the Wronskian of two functions $f(u)$ and $g(u)$

$$
w(f, g)=\frac{d f(u)}{d u} g(u)-f(u) \frac{d g(u)}{d u}
$$

In (2.25), $k_{22}(u)=1$ and we did not write the amplitude $k_{33}(u)$ but it is obtained from the amplitude $k_{11}(u)$ using the interchange rule (2.9).

Before we consider the type-II solution we observe that the parameters $\beta_{i j}$ are linked by the conditions (2.19) and (2.20) which together with the relation (2.18) and the normalization (2.6) imply that

$$
\text { if } \beta_{i j}=0 \text { than }\left\{\begin{array}{l}
k_{i j}(u)=0, \text { for } i \neq j \\
\text { or } \quad \text { for } i=j
\end{array}\right.
$$

It means that vanish free parameters in a general solution give us $K$-matrices with different forms defined by their non-vanishing entries. These reduced solutions will be presented in the appendices according to the values of $\beta_{13}$ and $\beta_{31}$, i.e., they are subclassified according to a of the four possibilities: (i) $\beta_{13} \neq 0$ and $\beta_{31} \neq 0$; (ii) $\beta_{13} \neq 0$ and $\beta_{31}=0$; (iii) $\beta_{13}=0$ and $\beta_{31} \neq 0$ and (iv) $\beta_{13}=0$ and $\beta_{31}=0$.

\subsection{Type-II solution}

For the type-II solution we have $\beta_{12}=\beta_{21}=\beta_{23}=\beta_{32}=0$. It means that the $K$-matrix has the form

$$
K_{I I}=\left(\begin{array}{ccc}
k_{11} & 0 & k_{13} \\
0 & 1 & 0 \\
k_{31} & 0 & k_{33}
\end{array}\right)
$$


Now the boundary YB equation (11.7) is composite of 28 reflection equations collected in eight blocks $B[1,3], B[1,5], B[1,7], B[2,4], B[2,6], B[3,5], B[3,7]$ and $B[4,6]$.

From the blocks

$$
B[2,4]=\left[\beta_{11} x_{2} y_{5}-2 w\left(x_{2}, y_{5}\right)\right] k_{11}+\beta_{31} x_{2} x_{5} k_{13}+\left[\beta_{11} x_{2} x_{5}+2 w\left(x_{2}, x_{5}\right)\right],
$$

and

$$
B[2,6]=\beta_{13} x_{2} y_{5} k_{11}+\left[\beta_{33} x_{2} x_{5}-2 w\left(x_{2}, x_{5}\right)\right] k_{13}+\beta_{13} x_{2} x_{5},
$$

we get the following four-parameter solution

$$
\begin{aligned}
& k_{11}=-\frac{\left[\beta_{11} x_{2} x_{5}+2 w\left(x_{2}, x_{5}\right)\right]\left[\beta_{33} x_{2} x_{5}-2 w\left(x_{2}, x_{5}\right)\right]-\beta_{13} \beta_{31} x_{2}^{2} x_{5}^{2}}{\left[\beta_{11} x_{2} y_{5}-2 w\left(x_{2}, y_{5}\right)\right]\left[\beta_{33} x_{2} x_{5}-2 w\left(x_{2}, x_{5}\right)\right]-\beta_{13} \beta_{31} x_{2}^{2} x_{5} y_{5}}, \\
& k_{33}=-\frac{\left[\beta_{11} x_{2} y_{5}+2 w\left(x_{2}, y_{5}\right)\right]\left[\beta_{33} x_{2} y_{5}-2 w\left(x_{2}, y_{5}\right)\right]-\beta_{13} \beta_{31} x_{2}^{2} y_{5}^{2}}{\left[\beta_{11} x_{2} y_{5}-2 w\left(x_{2}, y_{5}\right)\right]\left[\beta_{33} x_{2} x_{5}-2 w\left(x_{2}, x_{5}\right)\right]-\beta_{13} \beta_{31} x_{2}^{2} x_{5} y_{5}}, \\
& k_{13}=\frac{2 \beta_{13}\left[x_{2} x_{5} w\left(x_{2}, y_{5}\right)+x_{2} y_{5} w\left(x_{2}, x_{5}\right)\right]}{\left[\beta_{11} x_{2} y_{5}-2 w\left(x_{2}, y_{5}\right)\right]\left[\beta_{33} x_{2} x_{5}-2 w\left(x_{2}, x_{5}\right)\right]-\beta_{13} \beta_{31} x_{2}^{2} x_{5} y_{5}}, \\
& k_{31}=\frac{\beta_{31}}{\beta_{13}} k_{13} .
\end{aligned}
$$

Substituting (2.34) into the remaining blocks we will get constraint equations involving $\beta_{11}, \beta_{33}, \beta_{13}$ and $\beta_{31}$.

Having now built a common ground for all elements of the reflection matrix, we may proceed to find explicitly all regular $K$-matrices for each model.

We begin with the ZF model because of its simplicity and because all $K$-matrices are known from reference [24]. Doing this, besides confirming our result, we can compare its degree of simplicity.

\section{Regular K-matrix for the ZF model}

The simplest 19-vertex model is the ZF model. This vertex model is defined in terms of the Boltzmann weights given by the $R$-matrix of spin- 1 representation of $U_{q}\left(\widehat{s l}_{2}\right)$, or $A_{1}^{(1)}$ model. It is the trigonometric solution of the YB equation (11.1). $R(u)$ has the form (2.4) with non-zero entries [30]

$$
\begin{aligned}
& x_{1}(u)=\sinh (u+\eta) \sinh (u+2 \eta), \quad x_{2}(u)=\sinh u \sinh (u+\eta), \\
& x_{3}(u)=\sinh u \sinh (u-\eta), \quad x_{5}(u)=y_{5}(u)=\sinh (u+\eta) \sinh 2 \eta, \\
& x_{6}(u)=y_{6}(u)=\sinh u \sinh 2 \eta, \quad x_{7}(u)=y_{7}(u)=\sinh \eta \sinh 2 \eta, \\
& x_{4}(u)=x_{2}(u)+x_{7}(u) .
\end{aligned}
$$


In this model, the Wronskians (2.29) used in (2.25) and (2.34) are given by

$$
\begin{aligned}
& w\left(x_{1}, x_{2}\right)=-\sinh 2 \eta \sinh ^{2}(u+\eta), \\
& w\left(x_{2}, x_{5}\right)=w\left(x_{2}, y_{5}\right)=\cosh u \sinh 2 \eta \sinh ^{2}(u+\eta),
\end{aligned}
$$

and the $\Gamma$-functions $(2.26)$ have the form

$$
\Gamma_{1}(u)=\frac{\beta_{12} \sinh (u-\eta)-\beta_{23} \sinh u}{\sinh (2 u-\eta)}, \quad \Gamma_{2}(u)=\frac{\beta_{23} \sinh (u-\eta)-\beta_{12} \sinh u}{\sinh (2 u-\eta)} .
$$

Substituting these expressions into the constraint equation (2.21) we find in the first step that

$$
\sinh 2 \eta \sinh ^{2} u \sinh ^{3}(u+\eta) F(u, \eta)=0
$$

where

$$
\begin{aligned}
F(u, \eta)= & -2 \beta_{12}^{2} \beta_{23}^{2} \sinh \eta \sinh u \cosh (u-\eta)+\beta_{13}^{2} \beta_{21} \beta_{23} \sinh \eta \sinh 2 \eta \\
& -2 \beta_{12}^{2} \beta_{13} \sinh 2 \eta \cosh (2 u-\eta)+\beta_{12}^{2} \beta_{13} \beta_{33} \sinh 2 \eta \sinh (2 u-\eta) \\
& -2 \beta_{12} \beta_{13} \beta_{23} \sinh 2 \eta+\beta_{12} \beta_{13}^{2} \beta_{21} \sinh 2 \eta \sinh 2 u \\
& +\beta_{12}^{3} \beta_{23} \sinh \eta \sinh (2 u-2 \eta) .
\end{aligned}
$$

This equation should hold for all values of $u$. In particular, for $u=0$ we must have $F(0, \eta)=0$ from which we find $\beta_{21}$. Substituting $\beta_{21}$ into (3.5) we can still write $F(u, \eta)=\sinh u G(u, \eta)=0$. From the condition $G(0, \eta)=0$ we find $\beta_{33}$. Combining these results with the relations (2.19) and (2.20) we have

$$
\begin{aligned}
& \beta_{21}=\frac{\beta_{12}^{2} \beta_{23}}{\beta_{13}^{2}} \frac{1}{2 \cosh \eta}+\frac{\beta_{12}}{\beta_{13}} \frac{2}{\sinh \eta}, \\
& \beta_{33}=\frac{\beta_{12}^{2}}{\beta_{13}} \frac{1}{2 \cosh \eta}-2 \frac{\cosh \eta}{\sinh \eta}-\frac{\beta_{12} \beta_{23}}{\beta_{13}}, \\
& \beta_{11}=\frac{\beta_{23}^{2}}{\beta_{13}} \frac{1}{2 \cosh \eta}-2 \frac{\cosh \eta}{\sinh \eta}-\frac{\beta_{12} \beta_{23}}{\beta_{13}}, \\
& \beta_{31}=\frac{\beta_{21}^{2}}{\beta_{12}^{2}} \beta_{13}, \quad \beta_{32}=\frac{\beta_{21} \beta_{23}}{\beta_{12}} .
\end{aligned}
$$

The parameter $\beta_{11}$ is fixed by the interchange rule (2.9) or by direct computation with the constraint equation (2.22). These three free parameter expressions solve all constraint equations which come from the expressions for $k_{13}(u)$ discussed in the previous section.

Substituting (3.6) into the relations for type-I solution (2.25) we find the complete reflection $K$-matrix for the $\mathrm{ZF}$ model: 


$$
\begin{aligned}
k_{12}(u) & =\frac{1}{\beta_{13}} \Gamma_{1}(u) k_{13}(u), \quad k_{21}(u)=\frac{\beta_{21}}{\beta_{12} \beta_{13}} \Gamma_{1}(u) k_{13}(u), \\
k_{23}(u) & =\frac{1}{\beta_{13}} \Gamma_{2}(u) k_{13}(u), \quad k_{32}(u)=\frac{\beta_{21}}{\beta_{12} \beta_{13}} \Gamma_{2}(u) k_{13}(u), \\
k_{31}(u) & =\frac{\beta_{21}^{2}}{\beta_{12}^{2}} k_{13}(u), \\
k_{13}(u) & =\frac{\beta_{13}^{2} \sinh 2 \eta \sinh 2 u \sinh (2 u-\eta)}{\mathcal{D}(u, \eta)}, \\
k_{11}(u)= & \frac{1}{\mathcal{D}(u, \eta)}\left\{\sinh 2 \eta\left(\beta_{12} \beta_{23} \sinh u \sinh (u-\eta)-2 \beta_{13} \sinh (2 u-\eta)\right)\right. \\
& \left.-\sinh \eta \sinh u\left(\beta_{12}^{2} \sinh (u-\eta)+\beta_{23}^{2} \sinh (u+\eta)\right)\right\}, \\
k_{33}(u)= & \frac{1}{\mathcal{D}(u, \eta)}\left\{\sinh 2 \eta\left(\beta_{12} \beta_{23} \sinh u \sinh (u-\eta)-2 \beta_{13} \sinh (2 u-\eta)\right)\right. \\
& \left.-\sinh \eta \sinh u\left(\beta_{12}^{2} \sinh (u+\eta)+\beta_{23}^{2} \sinh (u-\eta)\right)\right\},
\end{aligned}
$$

where

$$
\begin{aligned}
\mathcal{D}(u, \eta)= & \beta_{12} \beta_{23}\left(\sinh \eta \sinh ^{2} 2 u+\sinh u \sinh (u-\eta) \sinh 2 \eta\right) \\
& -\left(\beta_{12}^{2}+\beta_{23}^{2}\right) \sinh u \sinh (u-\eta) \sinh \eta \\
& +4 \beta_{13} \cosh \eta \sinh (2 u-\eta) \sinh (2 u+\eta) .
\end{aligned}
$$

It is a regular solution of the RE equation (1.7) with three free parameters $\beta_{12}, \beta_{13}$ and $\beta_{32}$.

Using the relations

$$
\begin{aligned}
\beta_{12} & =-\frac{4 \mu \tilde{\mu} \sinh \left(\frac{\eta}{2}-\zeta\right) \cosh \eta}{2 \sinh \left(\frac{\eta}{2}+\zeta\right) \sinh \left(\frac{\eta}{2}-\zeta\right) \cosh \eta+\mu \tilde{\mu} \sinh ^{2} \eta} \\
\beta_{13} & =\frac{2 \mu^{2} \sinh \eta}{2 \sinh \left(\frac{\eta}{2}+\zeta\right) \sinh \left(\frac{\eta}{2}-\zeta\right) \cosh \eta+\mu \tilde{\mu} \sinh ^{2} \eta} \\
\beta_{32} & =\frac{4 \mu \tilde{\mu} \sinh \left(\frac{\eta}{2}+\zeta\right) \cosh \eta}{2 \sinh \left(\frac{\eta}{2}+\zeta\right) \sinh \left(\frac{\eta}{2}-\zeta\right) \cosh \eta+\mu \tilde{\mu} \sinh ^{2} \eta}
\end{aligned}
$$

where $\mu, \tilde{\mu}$ and $\zeta$ are new free parameters, we will reproduce the solution derived by Inami et al 24] using a different approach to solve the RE. 
Now we proceed to see the role of the type-II solution (2.30) in the ZF model. Taking into account the data of this model we substitute (2.30) into the equations $E[1,5]=0$ and $E[5,9]=0$ of the block $B[1,5]$ and write them in factored form

$$
2 \sinh ^{2} u \cosh u \sinh ^{4}(u+\eta) F_{i}(u, \eta)=0, \quad i=1,2
$$

where

$$
\begin{aligned}
F_{1}(u, \eta)= & 2 \beta_{11}(\sinh (u+3 \eta)-\cosh u \sinh \eta)-2 \beta_{33} \sinh u \cosh \eta \\
& +\left(\beta_{11} \beta_{33}-\beta_{13} \beta_{31}\right) \sinh u \sinh \eta+4 \cosh (u+3 \eta) \\
& +4 \cosh u \cosh \eta,
\end{aligned}
$$

and $F_{2}(u, \eta)$ is obtained from $F_{1}(u, \eta)$ by interchanging the indices $1 \leftrightarrow 3$. The conditions $F_{1}(0, \eta)=F_{2}(0, \eta)=0$ imply that

$$
\beta_{11}=\beta_{33}=-2 \frac{\cosh \eta}{\sinh \eta}
$$

If now we recall the relations (3.6) we will see that this is not a new solution but the one-parameter solution obtained by reduction of the type-I solution through the limit $\beta_{12} \rightarrow 0$ and $\beta_{23} \rightarrow 0$. The corresponding $K$ matrix has the form (2.31) with non-zero entries

$$
\begin{aligned}
k_{13}(u) & =\frac{1}{2} \frac{\beta_{13} \sinh \eta \sinh 2 u}{\sinh (2 u+\eta)}, \quad k_{31}(u)=\frac{1}{2} \frac{\beta_{31} \sinh \eta \sinh 2 u}{\sinh (2 u+\eta)} \\
k_{11}(u) & =k_{33}(u)=\frac{\sinh \eta}{\sinh (2 u-\eta)}, \\
\beta_{31} & =\frac{4}{\beta_{13} \sinh ^{2} \eta} .
\end{aligned}
$$

The relation between $\beta_{31}$ and $\beta_{13}$ in (3.13) was obtained using the equation $E[2,4]=0$.

In the appendix $\mathrm{A}$ we will present the remaining reduced solutions which can be obtained form the complete reflection $K$-matrix of the ZF model.

\section{Regular K-matrices for the IK model}

The solution of the YB equation corresponding to $A_{2}^{(2)}$ in the fundamental representation was found by Izergin and Korepin [32]. The $R$-matrix has the form (2.4) with non-zero entries

$$
x_{1}(u)=2 \sinh \left(\frac{u}{2}-2 \eta\right) \cosh \left(\frac{u}{2}-3 \eta\right), \quad x_{2}(u)=2 \sinh \frac{u}{2} \cosh \left(\frac{u}{2}-3 \eta\right),
$$




$$
\begin{aligned}
& x_{3}(u)=2 \sinh \frac{u}{2} \cosh \left(\frac{u}{2}-\eta\right), \\
& x_{4}(u)=2 \sinh \frac{u}{2} \cosh \left(\frac{u}{2}-3 \eta\right)-2 \sinh 2 \eta \cosh 3 \eta, \\
& x_{5}(u)=2 \mathrm{e}^{-\frac{1}{2} u} \sinh 2 \eta \cosh \left(\frac{u}{2}-3 \eta\right), \quad y_{5}(u)=-\mathrm{e}^{u} x_{5}(u), \\
& x_{6}(u)=2 \mathrm{e}^{-\frac{1}{2} u+2 \eta} \sinh 2 \eta \sinh \frac{u}{2}, \quad y_{6}(u)=\mathrm{e}^{u-4 \eta} x_{6}(u), \\
& x_{7}(u)=-2 \mathrm{e}^{-\frac{1}{2} u} \sinh 2 \eta\left[\cosh \left(\frac{u}{2}-3 \eta\right)+\mathrm{e}^{\eta} \sinh \frac{u}{2}\right], \\
& y_{7}(u)=-2 \mathrm{e}^{\frac{1}{2} u} \sinh 2 \eta\left[\cosh \left(\frac{u}{2}-3 \eta\right)-\mathrm{e}^{-\eta} \sinh \frac{u}{2}\right] .
\end{aligned}
$$

For this model the Wronskians are given by

$$
w\left(x_{1}, x_{2}\right)=2 \sinh 2 \eta \cosh ^{2}\left(\frac{u}{2}-3 \eta\right), w\left(x_{2}, x_{5}\right)=w\left(x_{2}, y_{5}\right)=-w\left(x_{1}, x_{2}\right) .
$$

A partial classification already exists for the reflection $K$-matrices of the IK model. Here we will use the procedure discussed in the section 2 to extend the results presented in 29.

Following the steps used in the construction of the solution for the ZF model, we start by considering the constraint equations (2.21) and (2.22) with the data of the IK model. After we find the expressions to $\beta_{21}$ and $\beta_{33}$ through the factorization procedure described in the section 2 , we will arrive at the following equation

$$
\left(\beta_{12}^{2}+\mathrm{e}^{4 \eta} \beta_{23}^{2}\right) \cosh 2 \eta \sinh \frac{u}{2}\left(\beta_{11} \mathrm{e}^{\frac{1}{2} u} \sinh \frac{u}{2}-1\right)=0
$$

which has an interchanged partner by the rule (2.10). Both equations must be valid for all values of $u$.

For $\beta_{12}, \beta_{23}, \beta_{21}$ and $\beta_{32}$ different of zero we have two solutions:

$$
\left\{\beta_{23}=i \mathrm{e}^{-2 \eta} \beta_{12}, \beta_{32}=i \mathrm{e}^{-2 \eta} \beta_{21}\right\} \quad \text { and } \quad\left\{\beta_{23}=-i \mathrm{e}^{-2 \eta} \beta_{12}, \beta_{32}=-i \mathrm{e}^{-2 \eta} \beta_{21}\right\} .
$$

The third solution is $\beta_{12}=\beta_{23}=\beta_{21}=\beta_{32}=0$.

\subsection{Type-I Solutions}

Due to (4.4), the constraint equations (2.21) and (2.22) for the IK model have two type-I solutions. Their parameters are given by

$$
\begin{aligned}
& \beta_{11}=\mp i \frac{\beta_{12}^{2}}{\beta_{13}} \frac{2 \mathrm{e}^{-2 \eta} \cosh \eta \pm i}{2 \cosh \eta} \mp i \mathrm{e}^{-\eta} \frac{1 \mp i \sinh \eta}{(\cosh \eta \mp i \sinh 2 \eta) \cosh \eta} \\
& \beta_{33}=\mp i \frac{\beta_{12}^{2}}{\beta_{13}} \frac{2 \mathrm{e}^{-4 \eta} \cosh \eta \mp i}{2 \cosh \eta} \mp i \mathrm{e}^{\eta} \frac{1 \mp i \sinh \eta}{(\cosh \eta \mp i \sinh 2 \eta) \cosh \eta}
\end{aligned}
$$




$$
\begin{aligned}
& \beta_{21}= \pm i \frac{\beta_{12}^{3}}{\beta_{13}^{2}} \frac{\mathrm{e}^{-2 \eta}}{2 \cosh \eta} \pm i \frac{\beta_{12}}{\beta_{13}} \frac{1 \mp i \sinh \eta}{(\cosh \eta \mp i \sinh 2 \eta) \cosh \eta} \\
& \beta_{23}= \pm i \beta_{12} \mathrm{e}^{-2 \eta}, \quad \beta_{32}= \pm i \beta_{21} \mathrm{e}^{-2 \eta}, \quad \beta_{31}=\frac{\beta_{21}^{2}}{\beta_{12}^{2}} \beta_{13}
\end{aligned}
$$

and their $\Gamma$-functions are given by

$$
\Gamma_{1}(u)=\beta_{12} \mathrm{e}^{-\frac{1}{2} u} \frac{\cosh \left(\frac{u}{2}-\eta\right) \pm i \sinh \left(\frac{u}{2}\right)}{\cosh (u-\eta)}, \quad \Gamma_{2}(u)= \pm i \mathrm{e}^{u-2 \eta} \Gamma_{1}(u)
$$

It means that these solutions have two free parameters, $\beta_{12}$ and $\beta_{13}$.

To write both type-I solutions of the IK model is a little bit cumbersome. Therefore, in order to save notation, we will only write one solution and take the conjugate of it to get the second solution.

For the first solution we find that the off-diagonal components of the $K$-matrix are given by

$$
\begin{aligned}
k_{12}(u) & =\frac{1}{\beta_{13}} \Gamma_{1}(u) k_{13}(u), \quad k_{21}(u)=\frac{\beta_{21}}{\beta_{12} \beta_{13}} \Gamma_{1}(u) k_{13}(u), \\
k_{23}(u) & =\frac{1}{\beta_{13}} \Gamma_{2}(u) k_{13}(u), \quad k_{32}(u)=\frac{\beta_{21}}{\beta_{12} \beta_{13}} \Gamma_{2}(u) k_{13}(u), \\
k_{31}(u) & =\frac{\beta_{21}^{2}}{\beta_{12}^{2}} k_{13}(u), \\
k_{13}(u) & =\frac{\beta_{13} \sinh u \cosh 3 \eta\left[\cosh \left(\frac{u}{2}-\eta\right)-i \sinh \frac{u}{2}\right]}{\cosh \eta\left[\cosh \left(\frac{u}{2}+3 \eta\right)-i \sinh \frac{u}{2}\right]+\cosh 3 \eta \sinh \frac{u}{2} \mathcal{F}(u, \eta)},
\end{aligned}
$$

and the diagonal components are given by

$$
\begin{aligned}
k_{11}(u) & =\frac{\mathrm{e}^{-u} \cosh \eta\left[\cosh \left(\frac{u}{2}-3 \eta\right)+i \sinh \frac{u}{2}\right]+\mathrm{e}^{-\frac{1}{2} u} \cosh 3 \eta \sinh \frac{u}{2} \Omega_{-}(u, \eta)}{\cosh \eta\left[\cosh \left(\frac{u}{2}+3 \eta\right)-i \sinh \frac{u}{2}\right]+\cosh 3 \eta \sinh \frac{u}{2} \mathcal{F}(u, \eta)}, \\
k_{33}(u) & =\frac{\mathrm{e}^{u} \cosh \eta\left[\cosh \left(\frac{u}{2}-3 \eta\right)+i \sinh \frac{u}{2}\right]-\mathrm{e}^{\frac{1}{2} u} \cosh 3 \eta \sinh \frac{u}{2} \Omega_{+}(u, \eta)}{\cosh \eta\left[\cosh \left(\frac{u}{2}+3 \eta\right)-i \sinh \frac{u}{2}\right]+\cosh 3 \eta \sinh \frac{u}{2} \mathcal{F}(u, \eta)}
\end{aligned}
$$

Here $\Omega_{\mp}(u, \eta)$ and $\mathcal{F}(u, \eta)$ are two-parameter functions defined by

$$
\begin{aligned}
\Omega_{\mp}(u, \eta) & =\left[\cosh (2 \eta) \cosh \left(\frac{u}{2}-\eta\right) \mp \frac{i}{2}\left(e^{\mp \frac{1}{2} u}+e^{ \pm \frac{1}{2} u} \cosh 2 \eta\right)\right] f(\eta), \\
\mathcal{F}(u, \eta) & =\left[\sinh \left(\frac{u}{2}-2 \eta\right) \cosh \left(\frac{u}{2}+\eta\right)+i \cosh ^{2}\left(\frac{u}{2}-\eta\right)\right] f(\eta),
\end{aligned}
$$

where

$$
f(\eta)=\frac{\beta_{12}^{2}}{\beta_{13}} \frac{\mathrm{e}^{-2 \eta}}{\cosh \eta}+\frac{2}{\cosh 2 \eta-i \sinh \eta} .
$$


The second type-I solution can be obtained from these expressions by interchanging $\pm i \leftrightarrow \mp i$. Of course there are many ways to write down these expressions. However, we chose to write them in this form in order to get more easily their reduced solutions in the appendix B.

\subsection{Type-II Solution}

The procedure to obtain type-I and type-II solutions are different by construction. To get the type-II we recall the expressions (2.34) with the data of the IK model. The result is a two-parameter $K$-matrix which has the form (2.31) with non-zero entries

$$
\begin{aligned}
& k_{31}(u)=\frac{\beta_{31}}{\beta_{13}} k_{13}(u), \\
& k_{13}(u)=\frac{\beta_{13} \sinh u}{1-2 \beta_{11} \mathrm{e}^{\eta} \sinh \frac{u}{2} \cosh \left(\frac{u}{2}-\eta\right)}, \\
& k_{11}(u)=\frac{1-2 \beta_{11} \mathrm{e}^{-\frac{1}{2} u+\eta} \sinh \frac{u}{2} \sinh \eta}{1-2 \beta_{11} \mathrm{e}^{\eta} \sinh \frac{u}{2} \cosh \left(\frac{u}{2}-\eta\right)}, \\
& k_{33}(u)=\frac{1+2 \beta_{11} \mathrm{e}^{\frac{1}{2} u+\eta} \sinh \frac{u}{2} \sinh \eta}{1-2 \beta_{11} \mathrm{e}^{\eta} \sinh \frac{u}{2} \cosh \left(\frac{u}{2}-\eta\right)} .
\end{aligned}
$$

Substituting (4.11) into the equation $E[1,5]=0$ we will find the following relations for the parameters

$$
\beta_{33}=\mathrm{e}^{2 \eta} \beta_{11} \quad \text { and } \quad \beta_{31}=\frac{1}{\beta_{13}} \mathrm{e}^{2 \eta} \beta_{11}^{2}
$$

Here we observe that this solution was already obtained in [29].

To summarize, we have obtained three regular general solutions with two free parameters of the RE for the IK model. The two first are type-I solution and the last is a type-II solution. If we compare the number of type-I solutions of the IK model with the number found for the ZF model we can see that the reduction of the number of free parameters from three for two increase the number of possible solutions as much as the possible manners of find the last parameter.

\section{$5 \quad$ Regular K-matrix for the $\operatorname{sl}(2 \mid 1)$ model}

The solution of the graded YB equation corresponding to $s l(2 \mid 1)$ in the fundamental representation has the form (2.4) with non-zero entries [5, 8, 31]:

$$
\begin{aligned}
& x_{1}(u)=\sinh (u+2 \eta) \cosh (u+\eta), \quad x_{2}(u)=\sinh u \cosh (u+\eta), \\
& x_{3}(u)=\sinh u \cosh (u-\eta), \quad x_{5}(u)=y_{5}(u)=\sinh 2 \eta \cosh (u+\eta),
\end{aligned}
$$




$$
\begin{aligned}
& x_{6}(u)=y_{6}(u)=\sinh u \sinh 2 \eta, \quad x_{7}(u)=y_{7}(u)=\sinh 2 \eta \cosh \eta, \\
& x_{4}(u)=x_{2}(u)-x_{7}(u) .
\end{aligned}
$$

For this model the Wronskians and the $\Gamma$-functions are given by

$$
\begin{gathered}
w\left(x_{1}, x_{2}\right)=-\sinh 2 \eta \cosh ^{2}(u+\eta), w\left(x_{2}, x_{5}\right)=\sinh 2 \eta \cosh u \cosh ^{2}(u+\eta), \\
\Gamma_{1}(u)=\frac{\beta_{12} \cosh (u-\eta)+\beta_{23} \sinh u}{\cosh (2 u-\eta)}, \Gamma_{2}(u)=\frac{\beta_{23} \cosh (u-\eta)-\beta_{12} \sinh u}{\cosh (2 u-\eta)} .
\end{gathered}
$$

From the similarity of these data with those of the ZF model, one can expect that the steps which we proceeded to solve the $s l(2 \mid 1)$-model are the same ones that we used in the ZF model. Therefore we need only to present the solution.

The parameters are given by

$$
\begin{aligned}
& \beta_{11}=-\frac{\beta_{23}^{2}}{\beta_{13}} \frac{1}{2 \sinh \eta}-2 \frac{\sinh \eta}{\cosh \eta}-\frac{\beta_{12} \beta_{23}}{\beta_{13}}, \\
& \beta_{33}=\frac{\beta_{12}^{2}}{\beta_{13}} \frac{1}{2 \sinh \eta}-2 \frac{\sinh \eta}{\cosh \eta}-\frac{\beta_{12} \beta_{23}}{\beta_{13}}, \\
& \beta_{21}=-\frac{\beta_{12}^{2} \beta_{23}}{\beta_{13}^{2}} \frac{1}{2 \sinh \eta}-\frac{\beta_{12}}{\beta_{13}} \frac{2}{\cosh \eta}, \\
& \beta_{31}=-\frac{\beta_{21}^{2}}{\beta_{12}^{2}} \beta_{13}, \quad \beta_{32}=-\frac{\beta_{21} \beta_{23}}{\beta_{12}},
\end{aligned}
$$

which results in a type-I solution with three free parameters. The off-diagonal elements are given by

$$
\begin{aligned}
k_{12}(u) & =\frac{1}{\beta_{13}} \Gamma_{1}(u) k_{13}(u), \quad k_{21}(u)=\frac{\beta_{21}}{\beta_{12} \beta_{13}} \Gamma_{1}(u) k_{13}(u), \\
k_{23}(u) & =\frac{1}{\beta_{13}} \Gamma_{2}(u) k_{13}(u), \quad k_{32}(u)=-\frac{\beta_{21}}{\beta_{12} \beta_{13}} \Gamma_{2}(u) k_{13}(u), \\
k_{31}(u) & =-\frac{\beta_{21}^{2}}{\beta_{12}^{2}} k_{13}(u), \\
k_{13}(u) & =\frac{\beta_{13}^{2} \sinh 2 \eta \sinh 2 u \cosh (2 u-\eta)}{\mathcal{D}(u, \eta)}
\end{aligned}
$$

and the diagonal elements are given by

$$
\begin{aligned}
k_{11}(u)= & \frac{1}{\mathcal{D}(u, \eta)}\left\{\sinh 2 \eta\left(-\beta_{12} \beta_{23} \sinh u \cosh (u-\eta)+2 \beta_{13} \cosh (2 u-\eta)\right)\right. \\
& \left.-\cosh \eta \sinh u\left(\beta_{12}^{2} \sinh (u-\eta)+\beta_{23}^{2} \cosh (u+\eta)\right)\right\}, \\
k_{33}(u)= & \frac{1}{\mathcal{D}(u, \eta)}\left\{\sinh 2 \eta\left(-\beta_{12} \beta_{23} \sinh u \cosh (u-\eta)+2 \beta_{13} \cosh (2 u-\eta)\right)\right. \\
& \left.+\cosh \eta \sinh u\left(\beta_{12}^{2} \cosh (u+\eta)+\beta_{23}^{2} \cosh (u-\eta)\right)\right\},
\end{aligned}
$$


where

$$
\begin{aligned}
\mathcal{D}(u, \eta)= & 2 \beta_{12} \beta_{23} \sinh u \cosh \eta(\sinh u+\cosh (2 u-\eta) \sinh (u+\eta)) \\
& +\left(\beta_{12}^{2}-\beta_{23}^{2}\right) \sinh u \cosh (u-\eta) \cosh \eta \\
& +4 \beta_{13} \sinh \eta \cosh (2 u-\eta) \cosh (2 u+\eta) .
\end{aligned}
$$

Regular reduced solutions from this complete reflection $K$-matrix are presented in the appendix C.

\section{Regular K-matrices for the $\operatorname{osp}(2 \mid 1)$ model}

The trigonometric solution of the graded YB equation for the fundamental representation of the $\operatorname{osp}(2 \mid 1)$ algebra was found by Bazhanov and Shadrikov in [8]. It has the form (2.4) with

$$
\begin{aligned}
& x_{1}(u)=\sinh (u+2 \eta) \sinh (u+3 \eta), \quad x_{2}(u)=\sinh u \sinh (u+3 \eta), \\
& x_{3}(u)=\sinh u \sinh (u+\eta), \\
& x_{4}(u)=\sinh u \sinh (u+3 \eta)-\sinh 2 \eta \sinh 3 \eta, \\
& x_{5}(u)=\mathrm{e}^{-u / 3} \sinh 2 \eta \sinh (u+3 \eta), \quad y_{5}(u)=\mathrm{e}^{u / 3} \sinh 2 \eta \sinh (u+3 \eta), \\
& x_{6}(u)=-\mathrm{e}^{-u / 3-2 \eta} \sinh 2 \eta \sinh u, \quad y_{6}(u)=\mathrm{e}^{u / 3+2 \eta} \sinh 2 \eta \sinh u, \\
& x_{7}(u)=\mathrm{e}^{u / 3} \sinh 2 \eta\left(\sinh (u+3 \eta)+\mathrm{e}^{-\eta} \sinh u\right), \\
& y_{7}(u)=\mathrm{e}^{-u / 3} \sinh 2 \eta\left(\sinh (u+3 \eta)+\mathrm{e}^{\eta} \sinh u\right) .
\end{aligned}
$$

For this model the Wronskians are given by

$$
\begin{aligned}
w\left(x_{1}, x_{2}\right) & =-\sinh 2 \eta \sinh ^{2}(u+3 \eta), \\
w\left(x_{2}, x_{5}\right) & =\frac{1}{3}\left(2 \mathrm{e}^{\frac{2}{3} u}+\mathrm{e}^{-\frac{4}{3} u}\right) \sinh 2 \eta \sinh ^{2}(u+3 \eta), \\
w\left(x_{2}, y_{5}\right) & =\frac{1}{3}\left(2 \mathrm{e}^{-\frac{2}{3} u}+\mathrm{e}^{\frac{4}{3} u}\right) \sinh 2 \eta \sinh ^{2}(u+3 \eta) .
\end{aligned}
$$

Now we will calculate the solutions of the RE for the $\operatorname{sep}(2 \mid 1)$ model. The procedure is a lot similar to that used to solve the IK model. The final constraint equations like (4.3) are

$$
\beta_{12}^{2}-\mathrm{e}^{-4 \eta} \beta_{23}^{2}=0 \quad \text { and } \quad \beta_{21}^{2}-\mathrm{e}^{-4 \eta} \beta_{32}^{2}=0 .
$$

For $\beta_{12}, \beta_{23}, \beta_{21}$ and $\beta_{32}$ different of zero we have two type-I solutions

$$
\left\{\beta_{23}=\mathrm{e}^{2 \eta} \beta_{12}, \beta_{32}=-\mathrm{e}^{2 \eta} \beta_{21}\right\} \quad \text { and } \quad\left\{\beta_{23}=-\mathrm{e}^{2 \eta} \beta_{12}, \beta_{32}=\mathrm{e}^{-2 \eta} \beta_{21}\right\} .
$$


The relative sign between $\beta_{23}$ and $\beta_{32}$ in both type-I solutions comes from the grading used. The third solution is $\beta_{12}=\beta_{23}=\beta_{21}=\beta_{32}=0$, the type-II solution.

\subsection{Type-I Solutions}

For the $\operatorname{osp}(2 \mid 1)$ model we have to write separately both type-I solutions. The first has the following relations among the parameters

$$
\begin{aligned}
& \beta_{11}=-\frac{\beta_{12}^{2}}{\beta_{13}} \frac{1+2 \mathrm{e}^{2 \eta} \sinh \eta}{2 \sinh \eta}+\frac{3 \mathrm{e}^{\eta}+4 \sinh \frac{1}{2} \eta \sinh \frac{3}{2} \eta}{3 \sinh \frac{1}{2} \eta \sinh \frac{3}{2} \eta} \\
& \beta_{33}=\frac{\beta_{23}^{2}}{\beta_{13}} \frac{1-2 \mathrm{e}^{-2 \eta} \sinh \eta}{2 \sinh \eta}-\frac{3 \mathrm{e}^{-\eta}+4 \sinh \frac{1}{2} \eta \sinh \frac{3}{2} \eta}{3 \sinh \frac{1}{2} \eta \sinh \frac{3}{2} \eta} \\
& \beta_{21}=\frac{\beta_{12}^{3}}{\beta_{13}^{2}} \frac{\mathrm{e}^{2 \eta}}{2 \sinh \eta}+\frac{\beta_{12}}{\beta_{13}} \frac{1}{\sinh \frac{1}{2} \eta \sinh \frac{3}{2} \eta} \\
& \beta_{23}=\mathrm{e}^{2 \eta} \beta_{12}, \quad \beta_{32}=-\mathrm{e}^{2 \eta} \beta_{21}, \quad \beta_{31}=-\frac{\beta_{21}^{2}}{\beta_{12}^{2}} \beta_{13} .
\end{aligned}
$$

The corresponding $\Gamma$-functions are given by

$$
\Gamma_{1}(u)=\beta_{12} e^{-\frac{1}{3} u} \frac{\sinh \frac{1}{2} \eta}{\sinh \frac{3}{2} \eta} \quad \text { and } \quad \Gamma_{2}(u)=\mathrm{e}^{\frac{2}{3} u+2 \eta} \Gamma_{1}(u) .
$$

Substituting these data into (2.25) we will have a complete reflection $K$-matrix with two free parameters. The corresponding entries are

$$
\begin{aligned}
k_{12}(u)= & \frac{1}{\beta_{13}} \Gamma_{1}(u) k_{13}(u), \quad k_{21}(u)=\frac{\beta_{21}}{\beta_{12} \beta_{13}} \Gamma_{1}(u) k_{13}(u), \\
k_{23}(u)= & \frac{1}{\beta_{13}} \Gamma_{2}(u) k_{13}(u), \quad k_{21}(u)=-\frac{\beta_{21}}{\beta_{12} \beta_{13}} \Gamma_{2}(u) k_{13}(u), \\
k_{13}(u)= & -\frac{1}{D_{1}(u, \eta)} \beta_{13}^{2} \sinh \frac{1}{2} \eta \sinh \eta \sinh \frac{3}{2} \eta \sinh \left(u+\frac{1}{2} \eta\right) \sinh 2 u, \\
k_{13}(u)= & -\frac{\beta_{21}^{2}}{\beta_{12}^{2}} k_{13}(u), \\
k_{11}(u)= & \frac{1}{\mathcal{D}_{1}(u, \eta)}\left\{\frac{1}{2} \mathrm{e}^{-\frac{2}{3} u} \sinh \left(u+\frac{1}{2} \eta\right)\left(\mathrm{e}^{2 u} \cosh 2 \eta-\cosh \eta\right) \mathcal{F}_{1}(u, \eta)\right. \\
& \left.-\beta_{12}^{2} \mathrm{e}^{-\frac{2}{3} u+2 \eta} \sinh ^{3} \frac{1}{2} \eta \sinh \frac{3}{2} \eta \sinh \left(u+\frac{3}{2} \eta\right)\right\}, \\
k_{33}(u)= & \frac{1}{\mathcal{D}_{1}(u, \eta)}\left\{\frac{1}{2} \mathrm{e}^{\frac{2}{3} u} \sinh ^{2}\left(u+\frac{1}{2} \eta\right)\left(\mathrm{e}^{-2 u} \cosh 2 \eta-\cosh \eta\right) \mathcal{F}_{1}(u, \eta)\right. \\
& \left.-\beta_{23}^{2} \mathrm{e}^{\frac{2}{3} u-2 \eta} \sinh ^{3} \frac{1}{2} \eta \sinh \frac{3}{2} \eta \sinh \left(u+\frac{3}{2} \eta\right)\right\},
\end{aligned}
$$


where

$$
\begin{aligned}
\mathcal{D}_{1}(u, \eta)= & \beta_{12}^{2} \mathrm{e}^{2 \eta} \sinh ^{3} \frac{1}{2} \eta \sinh \frac{3}{2} \eta \sinh \left(u-\frac{3}{2} \eta\right) \\
& -\sinh \left(u-\frac{1}{2} \eta\right) \sinh \left(u+\frac{1}{2} \eta\right) \sinh \left(u+\frac{3}{2} \eta\right) \mathcal{F}_{1}(u, \eta),
\end{aligned}
$$

and

$$
\mathcal{F}_{1}(u, \eta)=\beta_{12}^{2} \mathrm{e}^{2 \eta} \sinh \frac{1}{2} \eta \sinh \frac{3}{2} \eta-2 \beta_{13} \sinh \eta .
$$

For the second solution the parameters are given by

$$
\begin{aligned}
& \beta_{11}=-\frac{\beta_{12}^{2}}{\beta_{13}} \frac{1-2 \mathrm{e}^{2 \eta} \sinh \eta}{2 \sinh \eta}+\frac{3 \mathrm{e}^{\eta}-4 \cosh \frac{1}{2} \eta \cosh \frac{3}{2} \eta}{3 \cosh \frac{1}{2} \eta \cosh \frac{3}{2} \eta}, \\
& \beta_{33}=\frac{\beta_{23}^{2}}{\beta_{13}} \frac{1+2 \mathrm{e}^{-2 \eta} \sinh \eta}{2 \sinh \eta}+\frac{3 \mathrm{e}^{-\eta}-4 \cosh \frac{1}{2} \eta \cosh \frac{3}{2} \eta}{3 \cosh \frac{1}{2} \eta \cosh \frac{3}{2} \eta}, \\
& \beta_{21}=\frac{\beta_{12}^{3}}{\beta_{13}^{2}} \frac{\mathrm{e}^{2 \eta}}{2 \sinh \eta}-\frac{\beta_{12}}{\beta_{13}} \frac{1}{\cosh \frac{1}{2} \eta \cosh \frac{3}{2} \eta}, \\
& \beta_{23}=-\mathrm{e}^{2 \eta} \beta_{12}, \quad \beta_{32}=\mathrm{e}^{2 \eta} \beta_{21}, \quad \beta_{31}=-\frac{\beta_{21}^{2}}{\beta_{12}^{2}} \beta_{13},
\end{aligned}
$$

and we have new $\Gamma$-functions defined by

$$
\Gamma_{1}(u)=\beta_{12} e^{-\frac{1}{3} u} \frac{\cosh \frac{1}{2} \eta}{\cosh \frac{3}{2} \eta} \quad \text { and } \quad \Gamma_{2}(u)=-\mathrm{e}^{\frac{2}{3} u+2 \eta} \Gamma_{1}(u) .
$$

This results into a second complete reflection $K$-matrix with entries

$$
\begin{aligned}
k_{12}(u)= & \frac{1}{\beta_{13}} \Gamma_{1}(u) k_{13}(u), \quad k_{21}(u)=\frac{\beta_{21}}{\beta_{12} \beta_{13}} \Gamma_{1}(u) k_{13}(u), \\
k_{23}(u)= & \frac{1}{\beta_{13}} \Gamma_{2}(u) k_{13}(u), \quad k_{21}(u)=-\frac{\beta_{21}}{\beta_{12} \beta_{13}} \Gamma_{2}(u) k_{13}(u), \\
k_{13}(u)= & \frac{1}{\mathcal{D}_{2}(u, \eta)} \beta_{13}^{2} \cosh \frac{1}{2} \eta \cosh \frac{3}{2} \eta \cosh \left(u+\frac{1}{2} \eta\right) \sinh \eta \sinh 2 u, \\
k_{13}(u)= & -\frac{\beta_{21}^{2}}{\beta_{12}^{2}} k_{13}(u), \\
k_{11}(u)= & -\frac{1}{\mathcal{D}_{2}(u, \eta)}\left\{\frac{1}{2} \mathrm{e}^{-\frac{2}{3} u} \cosh \left(u+\frac{1}{2} \eta\right)\left(\mathrm{e}^{2 u} \cosh 2 \eta+\cosh \eta\right) \mathcal{F}_{2}(u, \eta)\right. \\
& \left.-\beta_{12}^{2} \mathrm{e}^{-\frac{2}{3} u+2 \eta} \cosh ^{3} \frac{1}{2} \eta \cosh \frac{3}{2} \eta \cosh \left(u+\frac{3}{2} \eta\right)\right\}, \\
k_{33}(u)= & -\frac{1}{\mathcal{D}_{2}(u, \eta)}\left\{\frac{1}{2} \mathrm{e}^{\frac{2}{3} u} \cosh \left(u+\frac{1}{2} \eta\right)\left(\mathrm{e}^{-2 u} \cosh 2 \eta+\cosh \eta\right) \mathcal{F}_{2}(u, \eta)\right. \\
& \left.-\beta_{23}^{2} \mathrm{e}^{\frac{2}{3} u-2 \eta} \cosh ^{3} \frac{1}{2} \eta \cosh \frac{3}{2} \eta \cosh \left(u+\frac{3}{2} \eta\right)\right\},
\end{aligned}
$$


where

$$
\begin{aligned}
\mathcal{D}_{2}(u, \eta)= & \beta_{12}^{2} \mathrm{e}^{2 \eta} \cosh ^{3} \frac{1}{2} \eta \cosh \frac{3}{2} \eta \cosh \left(u-\frac{3}{2} \eta\right) \\
& -\cosh \left(u-\frac{1}{2} \eta\right) \cosh \left(u+\frac{1}{2} \eta\right) \cosh \left(u+\frac{3}{2} \eta\right) \mathcal{F}_{2}(u, \eta),
\end{aligned}
$$

and

$$
\mathcal{F}_{2}(u, \eta)=\beta_{12}^{2} \mathrm{e}^{2 \eta} \cosh \frac{1}{2} \eta \cosh \frac{3}{2} \eta-2 \beta_{13} \sinh \eta
$$

\subsection{Type-II Solution}

The third solution is more simple. Now the parameters are related by

$$
\begin{aligned}
& \beta_{33}=-\mathrm{e}^{-2 \eta} \beta_{11}-\frac{8}{3} \mathrm{e}^{-\eta} \sinh \eta, \\
& \beta_{31}=-\frac{1}{\beta_{13}} \mathrm{e}^{-2 \eta}\left(\beta_{11}-\frac{4}{3}\right)^{2},
\end{aligned}
$$

and the corresponding $K$-matrix has the form (2.31) with the following non-zero entries

$$
\begin{aligned}
k_{31}(u) & =\frac{\beta_{31}}{\beta_{13}} k_{13}(u), \\
k_{13}(u) & =\frac{1}{2} \beta_{13} \frac{\sinh 2 u}{1-\left(\beta_{11}-\frac{4}{3}\right) \mathrm{e}^{-\eta} \sinh u \sinh (u+\eta)}, \\
k_{11}(u) & =\mathrm{e}^{-\frac{2}{3} u} \frac{1+2 e^{u} \sinh u+\left(\beta_{11}-\frac{4}{3}\right) \mathrm{e}^{u-\eta} \cosh \eta \sinh u}{1-\left(\beta_{11}-\frac{4}{3}\right) \mathrm{e}^{-\eta} \sinh u \sinh (u+\eta)}, \\
k_{33}(u) & =\mathrm{e}^{\frac{2}{3} u} \frac{1-2 e^{-u} \sinh u-\left(\beta_{11}-\frac{4}{3}\right) \mathrm{e}^{-u-\eta} \cosh \eta \sinh u}{1-\left(\beta_{11}-\frac{4}{3}\right) \mathrm{e}^{-\eta} \sinh u \sinh (u+\eta)} .
\end{aligned}
$$

The procedure to calculate (6.16) is the same ones used to calculate the type-II solution of the IK model. In the appendix D we present all reduced solutions for the $\operatorname{ssp}(2 \mid 1)$ model.

\section{Boundary Integrable Hamiltonians}

In order to derive the Hamiltonians it is convenient to expand the $R$-matrix around the regular point $u=0$. For 19-vertex models the corresponding solutions with the standard normalization can be read directly from (2.4). They have the form

$$
P R(u, \eta)=1+u\left(\alpha^{-1} h+\beta\right)+O\left(u^{2}\right)
$$

with $\alpha$ and $\beta$ being scalar functions. 
The two-site Hamiltonian $H_{k, k+1}$ (1.5) is the $h$ operator in (7.1) acting on the quantum spaces at sites $k$ and $k+1$. From (7.1) we obtain

$$
H_{k, k+1}=\left(\begin{array}{ccccccccc}
z_{1} & 0 & 0 & 0 & 0 & 0 & 0 & 0 & 0 \\
0 & \bar{z}_{5} & 0 & 1 & 0 & 0 & 0 & 0 & 0 \\
0 & 0 & \bar{z}_{7} & 0 & \bar{z}_{6} & 0 & z_{3} & 0 & 0 \\
0 & 1 & 0 & z_{5} & 0 & 0 & 0 & 0 & 0 \\
0 & 0 & \varepsilon \bar{z}_{6} & 0 & \varepsilon z_{4} & 0 & \varepsilon z_{6} & 0 & 0 \\
0 & 0 & 0 & 0 & 0 & \bar{z}_{5} & 0 & 1 & 0 \\
0 & 0 & z_{3} & 0 & z_{6} & 0 & z_{7} & 0 & 0 \\
0 & 0 & 0 & 0 & 0 & 1 & 0 & z_{5} & 0 \\
0 & 0 & 0 & 0 & 0 & 0 & 0 & 0 & z_{1}
\end{array}\right),
$$

which can be easily written in terms of the usual spin-1 operators:

$$
\begin{aligned}
H_{k, k+1}= & \epsilon z_{4}+\frac{1}{2}\left(\bar{z}_{5}-z_{5}\right)\left[S_{k}^{z}-S_{k+1}^{z}\right]+\frac{1}{2}\left(z_{5}+\bar{z}_{5}-2 \epsilon z_{4}\right)\left[\left(S_{k}^{z}\right)^{2}+\left(S_{k+1}^{z}\right)^{2}\right] \\
& +\frac{1}{4}\left(2 z_{1}-z_{7}-\bar{z}_{7}\right) S_{k}^{z} S_{k+1}^{z}+\frac{1}{4}\left[2\left(z_{1}-z_{5}-\bar{z}_{5}\right)+x_{7}+\bar{z}_{7}+4 \epsilon z_{4}\right]\left(S_{k}^{z} S_{k+1}^{z}\right)^{2} \\
& +\frac{1}{4}\left(z_{7}-\bar{z}_{7}-2\left(x_{5}-\bar{z}_{5}\right)\right)\left[\left(S_{k}^{z}\right)^{2} S_{k+1}^{z}-S_{k}^{z}\left(S_{k+1}^{z}\right)^{2}\right] \\
& +\frac{1}{4} z_{3}\left[\left(S_{k}^{+} S_{k+1}^{-}\right)^{2}+\left(S_{k}^{-} S_{k+1}^{+}\right)^{2}\right]-\frac{1}{2}\left[\epsilon z_{6} S_{k}^{+} S_{k+1}^{-}+\epsilon \bar{z}_{6} S_{k}^{-} S_{k+1}^{+}\right] S_{k}^{z} S_{k+1}^{z} \\
& -\frac{1}{2} S_{k}^{z} S_{k+1}^{z}\left[\bar{z}_{6} S_{k}^{+} S_{k+1}^{-}+z_{6} S_{k}^{-} S_{k+1}^{+}\right]+\frac{1}{2}\left\{S_{k}^{+} S_{k}^{z} S_{k+1}^{z} S_{k+1}^{-}+S_{k}^{-} S_{k}^{z} S_{k+1}^{z} S_{k+1}^{+}\right. \\
& \left.+S_{k}^{z} S_{k}^{+} S_{k+1}^{-} S_{k+1}^{z}+S_{k}^{z} S_{k}^{-} S_{k+1}^{+} S_{k+1}^{z}\right\}
\end{aligned}
$$

where

$$
\begin{aligned}
\varepsilon & =1, \quad \alpha=\sinh 2 \eta, \quad \beta=0, \quad z_{1}=0, \quad z_{3}=-1, \quad z_{4}=-2 \cosh 2 \eta \\
z_{5} & =\bar{z}_{5}=-\cosh 2 \eta, \quad z_{6}=\bar{z}_{6}=2 \cosh \eta, \quad z_{7}=\bar{z}_{7}=-1-2 \cosh 2 \eta
\end{aligned}
$$

for the ZF model;

$$
\begin{gathered}
\varepsilon=-1, \quad \alpha=\sinh 2 \eta, \quad \beta=0 \quad z_{1}=0, \quad z_{3}=1, \quad z_{4}=2 \cosh 2 \eta, \\
\bar{z}_{5}=z_{5}=-\cosh 2 \eta, \quad \bar{z}_{6}=z_{6}=2 \sinh \eta, \quad \bar{z}_{7}=z_{7}=-1-4 \sinh ^{2} \eta
\end{gathered}
$$

for the $\operatorname{sl}(2 \mid 1)$ model;

$$
\begin{aligned}
\varepsilon & =1, \alpha=-2 \sinh 2 \eta, \beta=0, z_{1}=0, z_{3}=\frac{\cosh \eta}{\cosh 3 \eta}, z_{4}=-2 \frac{\sinh 4 \eta \sinh \eta}{\cosh 3 \eta} \\
z_{5} & =-\mathrm{e}^{-2 \eta}, \bar{z}_{5}=-\mathrm{e}^{2 \eta}, z_{6}=\mathrm{e}^{2 \eta} \frac{\sinh 2 \eta}{\cosh 3 \eta}, \bar{z}_{6}=-\mathrm{e}^{-2 \eta} \frac{\sinh 2 \eta}{\cosh 3 \eta} \\
z_{7} & =-\mathrm{e}^{-4 \eta} \frac{\cosh \eta}{\cosh 3 \eta}, \bar{z}_{7}=-\mathrm{e}^{4 \eta} \frac{\cosh \eta}{\cosh 3 \eta}
\end{aligned}
$$


for the IK model and

$$
\begin{aligned}
\varepsilon & =-1, \alpha=\sinh 2 \eta, \beta=-\operatorname{coth} 2 \eta, z_{1}=\cosh 2 \eta, z_{3}=\frac{\sinh \eta}{\sinh 3 \eta}, \\
z_{4} & =1+\operatorname{coth} 3 \eta \sinh 2 \eta, z_{5}=-\frac{\sinh 2 \eta}{3}, \bar{z}_{5}=-z_{5}, z_{6}=-\mathrm{e}^{-2 \eta} \frac{\sinh 2 \eta}{\sinh 3 \eta} \\
\bar{z}_{6} & =\mathrm{e}^{2 \eta} \frac{\sinh 2 \eta}{\sinh 3 \eta}, z_{7}=\frac{\sinh 2 \eta}{3}+\mathrm{e}^{-\eta} \frac{\sinh 2 \eta}{\sinh 3 \eta}, \bar{z}_{7}=-\frac{\sinh 2 \eta}{3}+\mathrm{e}^{\eta} \frac{\sinh 2 \eta}{\sinh 3 \eta}
\end{aligned}
$$

for the $\operatorname{osp}(2 \mid 1)$ model.

Next, we recall (1.6) to derive the boundary terms which can now be read from the $K$-matrices obtained in this paper. Due to (2.7), the first boundary term is easily obtained:

$$
\left.\frac{d K_{-}(u)}{d u}\right|_{u=0}=\left(\begin{array}{ccc}
\beta_{11} & \beta_{12} & \beta_{13} \\
\beta_{21} & 0 & \beta_{23} \\
\beta_{31} & \beta_{32} & \beta_{33}
\end{array}\right)
$$

where $\beta_{i j}$ are the parameters calculated in the previous sections for the general solutions of the 19-vertex models considered in this paper. Therefore the integrable spin-1 Hamiltonians with general boundary interactions associated with these vertex models can be write in the form

$$
H=\sum_{k=1}^{N-1} H_{k, k+1}+\frac{1}{2} \sum_{i, j=1}^{3} \beta_{i j} \mathrm{E}_{i j}^{(1)} \otimes 1+\frac{1}{2} \sum_{i, j=1}^{3} \alpha_{i j} 1 \otimes \mathrm{E}_{i j}^{(N)}
$$

where $\mathrm{E}_{i j}$ is an $3 \times 3$ matrix with only non-vanishing entry 1 in row $i$ and column $j$. $\alpha_{i j}$ are new parameters associated with the left $K$-matrices, obtained from the second boundary term $\operatorname{tr}_{0} \stackrel{0}{K}+(0) H_{N, 0} / \operatorname{tr} K_{+}(0)$ through the correspondence (1.8), i.e.

$$
K_{-}\left(u, \beta_{i j}\right) \longrightarrow K_{+}\left(u, \alpha_{j i}\right)=K_{-}^{t}\left(-u-\rho, \alpha_{j i}\right) M
$$

For the graded models the same results follow after we use the graded formulation for (7.9) and (7.10).

\section{Conclusion}

We have considered the boundary YB equation for some 19-vertex models. After a systematic study of the functional equations we find that there is only a complete solution with three free parameters for the $\mathrm{ZF}$ and $\operatorname{sl}(2 \mid 1)$ models. From vanishing of these parameters we can derive some particular solutions, which we called reduced solutions. In these models (see appendices A and B) the last possible reduction will give us a diagonal solution with one free parameter. 
For IK and $\operatorname{sep}(2 \mid 1)$ models we find two complete solutions but with only two free parameters. This decrease in the number of free parameters is responsible for the appearance of a third solution, the type-II solution, which also have two free parameters but with some vanishing entries. From these general solutions we find reduced solutions by vanishing of their free parameters (see appendices $\mathrm{C}$ and $\mathrm{D}$ ). The last possible reduction for these models gives us three diagonal solutions with no free parameters. This explains the result obtained for the IK model in a previous work [34].

Acknowledgment: This work was supported in part by Fundação de Amparo à Pesquisa do Estado de São Paulo-FAPESP-Brasil and by Conselho Nacional de Desenvolvimento-CNPq-Brasil.

\section{Appendix A. ZF Reduced Solutions}

In this appendix we will present particular solutions of the RE for the ZF model. These solutions are obtained by vanishing some free parameters of the general solution

derived in the section 3 . Following the sub-classification discussed in the section 2 we have

Case (i) $\beta_{13} \neq 0$ and $\beta_{31} \neq 0$ : The reduced solution in this case was presented in the section 3 . It is given by (3.13).

Case (ii) $\beta_{13} \neq 0$ and $\beta_{31}=0$ : In this case we have two regular solutions

$$
K_{I_{b}}=\left(\begin{array}{ccc}
k_{11} & k_{12} & k_{13} \\
0 & 1 & k_{23} \\
0 & 0 & k_{33}
\end{array}\right) \quad \text { and } \quad K_{I_{c}}=\left(\begin{array}{ccc}
k_{11} & 0 & k_{13} \\
0 & 1 & 0 \\
0 & 0 & k_{33}
\end{array}\right)
$$

The parameters for the solution $K_{I_{b}}$ are obtained by taking the limit $\beta_{21} \rightarrow 0$ in (3.6). From this limit we have the following parameters

$$
\begin{aligned}
\beta_{12} & =-\frac{1}{2} \beta_{23}\left(\beta_{11} \sinh \eta-2 \cosh \eta\right) \\
\beta_{33} & =2 \frac{\beta_{11} \cosh \eta-2 \sinh \eta}{\beta_{11} \sinh \eta-2 \cosh \eta} \\
\beta_{13} & =\frac{1}{8} \frac{\beta_{23}^{2} \sinh \eta\left(\beta_{11} \sinh \eta-2 \cosh \eta\right)}{\cosh \eta} \\
\beta_{11} & \neq 2 \operatorname{coth} \eta
\end{aligned}
$$

Substituting (A2) into (3.7) we find

$$
k_{12}(u)=\frac{1}{2} \frac{\beta_{23}\left(\beta_{11} \sinh \eta-2 \cosh \eta\right) \sinh 2 u}{\beta_{11} \sinh u-2 \cosh (u)}
$$




$$
\begin{gathered}
k_{23}(u)=-\frac{1}{2} \frac{\beta_{23}\left(\beta_{11} \sinh \eta-2 \cosh \eta\right) \sinh 2 u}{\beta_{11} \sinh (u-\eta)+2 \cosh (u-\eta)} \\
k_{13}(u)=-\frac{\beta_{13}\left(\beta_{11} \sinh \eta-2 \cosh \eta\right) \sinh 2 u \sinh (2 u-\eta)}{\sinh \eta\left(\beta_{11} \sinh u-2 \cosh u\right)\left(\beta_{11} \sinh (u-\eta)+2 \cosh (u-\eta)\right)} \\
k_{11}(u)=-\frac{\beta_{11} \sinh u+2 \cosh u}{\beta_{11} \sinh u-2 \cosh u} \\
k_{33}(u)=-\frac{\beta_{11} \sinh (u+\eta)-2 \cosh (u+\eta)}{\beta_{11} \sinh (u-\eta)+2 \cosh (u-\eta)}
\end{gathered}
$$

Note that we have choose to write the parameters in terms of $\beta_{11}$ and $\beta_{23}$. The solution $K_{I_{c}}$ is the limit $\beta_{23} \rightarrow 0$ of $K_{I_{b}}$. In this case there is no relation between $\beta_{11}$ and $\beta_{13}$ but, using the equation $E[1,3]=0$ one can see that it is a two-parameter solution provided that

$$
\sinh 4 \eta=0, \quad \sinh 2 \eta \neq 0 \quad \text { and } \quad \beta_{11} \neq 2 \operatorname{coth} \eta
$$

Case (iii) $\beta_{13}=0$ and $\beta_{31} \neq 0$ : This case is a transposition of the previous one. The corresponding $K$-matrices are

$$
K_{I_{d}}=\left(\begin{array}{ccc}
k_{11} & 0 & 0 \\
k_{21} & 1 & 0 \\
k_{31} & k_{32} & k_{33}
\end{array}\right) \quad \text { and } \quad K_{I_{e}}=\left(\begin{array}{ccc}
k_{11} & 0 & 0 \\
0 & 1 & 0 \\
k_{31} & 0 & k_{33}
\end{array}\right)
$$

Their parameters and non-zero entries are obtained from (A.2) and (A.3) respectively, using the interchange rule (2.10). For $K_{I_{e}}$ we have the condition (A.4) still.

Case (iv) $\beta_{13}=0$ and $\beta_{31}=0$ : Finally we arrive at the diagonal solution. It has the form

$$
K_{I_{f}}=\left(\begin{array}{ccc}
k_{11} & 0 & 0 \\
0 & 1 & 0 \\
0 & 0 & k_{33}
\end{array}\right)
$$

which is an one-parameter solution with

$$
\begin{aligned}
k_{11}(u) & =-\frac{\beta_{11} \sinh u+2 \cosh u}{\beta_{11} \sinh u-2 \cosh u} \\
k_{33}(u) & =-\frac{\beta_{11} \sinh (u+\eta)-2 \cosh (u+\eta)}{\beta_{11} \sinh (u-\eta)+2 \cosh (u-\eta)} .
\end{aligned}
$$

It is obtained, for instance, from the solution $K_{I_{b}}$ when $\beta_{23} \rightarrow 0$. This diagonal solution was derived in 33. 


\section{Appendix B. IK Reduced Solutions}

For the IK model we have three general solutions. Therefore, we will find three types of reduced solutions. Reduced solutions from the type-I solutions discussed in the section 4 are here obtained using the same limit procedure presented in the ZF model

Case (i) $\beta_{13} \neq 0$ and $\beta_{31} \neq 0$ we have two one-parameter solutions

$$
K_{I_{a}}=\left(\begin{array}{ccc}
k_{11} & 0 & k_{13} \\
0 & 1 & 0 \\
k_{31} & 0 & k_{33}
\end{array}\right)
$$

where

$$
\begin{aligned}
& k_{11}(u)=\frac{\cosh 2 \eta \mp i \mathrm{e}^{-u} \sinh \eta}{\cosh 2 \eta \pm i \sinh (u-\eta)}, k_{33}(u)=\frac{\cosh 2 \eta \mp i \mathrm{e}^{u} \sinh \eta}{\cosh 2 \eta \pm i \sinh (u-\eta)} \\
& k_{13}(u)=\frac{\beta_{13}(\cosh 2 \eta \mp i \sinh \eta) \sinh u}{\cosh 2 \eta \pm i \sinh (u-\eta)}, k_{31}(u)=\frac{\beta_{31}}{\beta_{13}} k_{13}(u)
\end{aligned}
$$

with the relation

$$
\beta_{13} \beta_{31}(\cosh 2 \eta \mp i \sinh \eta)^{2}=-1
$$

Here we observe that these reduced solutions can be derived from the type-II solution. Substituting

$$
\beta_{11}=\mp \frac{i e^{-\eta}}{\cosh 2 \eta \mp i \sinh \eta}
$$

into (4.11) we will get (B.2).

Case (ii) $\beta_{13} \neq 0$ and $\beta_{31}=0$ : Here we have four reduced solutions

$$
K_{I_{b}}=\left(\begin{array}{ccc}
k_{11} & k_{12} & k_{13} \\
0 & 1 & k_{23} \\
0 & 0 & k_{33}
\end{array}\right) \quad \text { and } \quad K_{I_{c}}=\left(\begin{array}{ccc}
k_{11} & 0 & k_{13} \\
0 & 1 & 0 \\
0 & 0 & k_{33}
\end{array}\right)
$$

where

$$
\begin{aligned}
\beta_{12}^{2} & =-2 \mathrm{e}^{2 \eta} \beta_{13} \frac{\cosh \eta}{\cosh 2 \eta \mp i \sinh \eta} \\
\Gamma_{1}(u) & =\beta_{12} \mathrm{e}^{-\frac{1}{2} u} \frac{\cosh \left(\frac{u}{2}-\eta\right) \pm i \sinh \frac{u}{2}}{\cosh (u-\eta)} \\
k_{12}(u) & =\frac{1}{\beta_{13}} \Gamma_{1}(u) k_{13}(u), k_{23}(u)=\frac{ \pm i \mathrm{e}^{u-2 \eta}}{\beta_{13}} \Gamma_{1}(u) k_{13}(u) \\
k_{13}(u) & =\beta_{13} \frac{\cosh 3 \eta \sinh u}{\cosh \eta} \frac{\cosh \left(\frac{u}{2}-\eta\right) \mp i \sinh \frac{u}{2}}{\cosh \left(\frac{u}{2}+3 \eta\right) \mp i \sinh \frac{u}{2}} \\
k_{11}(u) & =\mathrm{e}^{-u} \frac{\cosh \left(\frac{u}{2}-3 \eta\right) \pm i \sinh \frac{u}{2}}{\cosh \left(\frac{u}{2}+3 \eta\right) \mp i \sinh \frac{u}{2}} \\
k_{33}(u) & =\mathrm{e}^{u} \frac{\cosh \left(\frac{u}{2}-3 \eta\right) \pm i \sinh \frac{u}{2}}{\cosh \left(\frac{u}{2}+3 \eta\right) \mp i \sinh \frac{u}{2}}
\end{aligned}
$$


The solutions $K_{I_{c}}$ are one-parameter solutions provided that

$$
\sinh 2 \eta=0 \quad \text { and } \quad \sinh \eta \neq 0 .
$$

Case (iii) $\beta_{13}=0$ and $\beta_{31} \neq 0$ : In this case we have four solutions

$$
K_{I_{d}}=\left(\begin{array}{ccc}
k_{11} & 0 & 0 \\
k_{21} & 1 & 0 \\
k_{31} & k_{32} & k_{33}
\end{array}\right) \quad \text { and } \quad K_{I_{e}}=\left(\begin{array}{ccc}
k_{11} & 0 & 0 \\
0 & 1 & 0 \\
k_{31} & 0 & k_{33}
\end{array}\right)
$$

they are obtained from (B.6) by the interchange $k_{i j} \leftrightarrow k_{j i}$ and $\beta_{i j} \leftrightarrow \beta_{j i}$.

Case (iv) $\beta_{13}=0$ and $\beta_{31}=0$ : In this case we have two diagonal solutions with no free parameters

$$
K_{I_{f}}=\left(\begin{array}{ccc}
k_{11} & 0 & 0 \\
0 & 1 & 0 \\
0 & 0 & k_{33}
\end{array}\right)
$$

where

$$
k_{11}(u)=\mathrm{e}^{-u} \frac{\cosh \left(\frac{u}{2}-3 \eta\right) \pm i \sinh \frac{u}{2}}{\cosh \left(\frac{u}{2}+3 \eta\right) \mp i \sinh \frac{u}{2}}, k_{33}(u)=\mathrm{e}^{u} \frac{\cosh \left(\frac{u}{2}-3 \eta\right) \pm i \sinh \frac{u}{2}}{\cosh \left(\frac{u}{2}+3 \eta\right) \mp i \sinh \frac{u}{2}}
$$

Moreover, from the type-II solution (4.11) we have three more reduced solutions. The two first are one-parameter solutions

$$
K_{I I_{a}}=\left(\begin{array}{ccc}
1 & 0 & \beta_{13} \sinh u \\
0 & 1 & 0 \\
0 & 0 & 1
\end{array}\right) \quad \text { and } \quad K_{I I_{b}}=\left(\begin{array}{ccc}
1 & 0 & 0 \\
0 & 1 & 0 \\
\beta_{31} \sinh u & 0 & 1
\end{array}\right)
$$

and the last is the trivial solution

$$
K_{I I_{c}}=\left(\begin{array}{ccc}
1 & 0 & 0 \\
0 & 1 & 0 \\
0 & 0 & 1
\end{array}\right)
$$

They belong to the cases (ii), (iii) and (iv), respectively. The diagonal solutions (B.9) and (B.12) were obtained for the first time by Mezincescu and Nepomechie [34]. 


\section{Appendix C. $s l(2 \mid 1)$ Reduced Solutions}

In this appendix we present the reduced solution for the $\operatorname{sl}(2 \mid 1)$ model. They are obtained by the vanishing of some parameters in the general solution derived in section 5. The limit procedure is the same used for the ZF model:

Case (i) $\beta_{13} \neq 0$ and $\beta_{31} \neq 0$ : The corresponding $K$-matrix has the form

$$
K_{I_{a}}=\left(\begin{array}{ccc}
k_{11} & 0 & k_{13} \\
0 & 1 & 0 \\
k_{31} & 0 & k_{33}
\end{array}\right)
$$

where

$$
\begin{aligned}
k_{13}(u) & =\frac{1}{2} \frac{\beta_{13} \cosh \eta \sinh 2 u}{\cosh (2 u+\eta)}, \quad k_{31}(u)=-\frac{2 \sinh 2 u}{\beta_{13} \cosh \eta \cosh (2 u+\eta)} \\
k_{11}(u) & =k_{33}(u)=\frac{\cosh \eta}{\cosh (2 u-\eta)}
\end{aligned}
$$

It is a one-parameter solution of the graded RE.

Case (ii) $\beta_{13} \neq 0$ and $\beta_{31}=0$ : Here we have two reduced solutions with two free parameters

$$
K_{I_{b}}=\left(\begin{array}{ccc}
k_{11} & k_{12} & k_{13} \\
0 & 1 & k_{23} \\
0 & 0 & k_{33}
\end{array}\right) \quad \text { and } \quad K_{I_{c}}=\left(\begin{array}{ccc}
k_{11} & 0 & k_{13} \\
0 & 1 & 0 \\
0 & 0 & k_{33}
\end{array}\right)
$$

where

$$
\begin{aligned}
k_{13}(u) & =-\frac{\beta_{13}\left(\beta_{11} \cosh \eta-2 \sinh \eta\right) \sinh 2 u \cosh (2 u-\eta)}{\cosh \eta\left(\beta_{11} \sinh u-2 \cosh u\right)\left(\beta_{11} \cosh (u-\eta)+2 \sinh (u-\eta)\right)} \\
k_{12}(u) & =-\frac{1}{2} \frac{\beta_{23}\left(\beta_{11} \cosh \eta-2 \sinh \eta\right) \sinh 2 u}{\beta_{11} \sinh u-2 \cosh (u)} \\
k_{23}(u) & =\frac{1}{2} \frac{\beta_{23}\left(\beta_{11} \cosh \eta-2 \sinh \eta\right) \sinh 2 u}{\beta_{11} \cosh (u-\eta)+2 \sinh (u-\eta)} \\
k_{11}(u) & =-\frac{\beta_{11} \sinh u+2 \cosh u}{\beta_{11} \sinh u-2 \cosh u} \\
k_{33}(u) & =\frac{\beta_{11} \cosh (u+\eta)-2 \sinh (u+\eta)}{\beta_{11} \cosh (u-\eta)+2 \sinh (u-\eta)}
\end{aligned}
$$

with the following relations for the parameters

$$
\beta_{13}=-\frac{1}{8} \frac{\beta_{23}^{2} \cosh \eta\left(\beta_{11} \cosh \eta-2 \sinh \eta\right)}{\sinh \eta}, \quad \beta_{11} \neq 2 \tanh \eta
$$

The parameter $\eta$ in the solution $K_{I_{c}}$ is solution of the equation:

$$
\sinh 4 \eta=0 \quad \text { and } \quad \sinh 2 \eta \neq 0 .
$$


Case (iii) $\beta_{13}=0$ and $\beta_{31} \neq 0$ : This is a super-transposition of the previous case

$$
K_{I_{d}}=\left(\begin{array}{ccc}
k_{11} & 0 & 0 \\
k_{21} & 1 & 0 \\
k_{31} & k_{32} & k_{33}
\end{array}\right) \quad \text { and } \quad K_{I_{e}}=\left(\begin{array}{ccc}
k_{11} & 0 & 0 \\
0 & 1 & 0 \\
k_{31} & 0 & k_{33}
\end{array}\right)
$$

with matrix elements and parameters obtained from (C.4) and (C.5) respectively, through the interchange $k_{i j} \leftrightarrow k_{j i}$ and $\beta_{i j} \leftrightarrow \beta_{j i}$.

Case (iv) $\beta_{13}=0$ and $\beta_{31}=0$ : In yhis case we have a one-parameter diagonal solution

$$
K_{I_{f}}=\left(\begin{array}{ccc}
k_{11} & 0 & 0 \\
0 & 1 & 0 \\
0 & 0 & k_{33}
\end{array}\right)
$$

where

$$
\begin{aligned}
k_{11}(u) & =-\frac{\beta_{11} \sinh u+2 \cosh u}{\beta_{11} \sinh u-2 \cosh u} \\
k_{33}(u) & =\frac{\beta_{11} \cosh (u+\eta)-2 \sinh (u+\eta)}{\beta_{11} \cosh (u-\eta)+2 \sinh (u-\eta)}
\end{aligned}
$$

This diagonal solution was already known [23]. 


\section{Appendix D. $\operatorname{osp}(2 \mid 1)$ Reduced Solutions}

In this appendix we will present the three types of reduced solutions for the $\operatorname{ssp}(2 \mid 1)$ model

Case (i) $\beta_{13} \neq 0$ and $\beta_{31} \neq 0$ : In this case we have two one-parameter solutions

$$
K_{I_{a}}=\left(\begin{array}{ccc}
k_{11} & 0 & k_{13} \\
0 & 1 & 0 \\
k_{31} & 0 & k_{33}
\end{array}\right)
$$

where

$$
\begin{aligned}
k_{11}(u) & =\frac{1}{2} \mathrm{e}^{-\frac{2}{3} u} \frac{\cosh \eta-\mathrm{e}^{2 u} \cosh 2 \eta}{\sinh \left(u-\frac{1}{2} \eta\right) \sinh \left(u+\frac{3}{2} \eta\right)} \\
k_{33}(u) & =\frac{1}{2} \mathrm{e}^{\frac{2}{3} u} \frac{\cosh \eta-\mathrm{e}^{-2 u} \cosh 2 \eta}{\sinh \left(u-\frac{1}{2} \eta\right) \sinh \left(u+\frac{3}{2} \eta\right)} \\
k_{13}(u) & =-\frac{1}{2} \frac{\beta_{13} \sinh \frac{1}{2} \eta \sinh \frac{3}{2} \eta \sinh 2 u}{\sinh \left(u-\frac{1}{2} \eta\right) \sinh \left(u+\frac{3}{2} \eta\right)} \\
k_{31}(u) & =\frac{1}{2} \frac{\sinh 2 u}{\beta_{13} \sinh \frac{1}{2} \eta \sinh \frac{3}{2} \eta \sinh \left(u-\frac{1}{2} \eta\right) \sinh \left(u+\frac{3}{2} \eta\right)}
\end{aligned}
$$

for the first solution and

$$
\begin{aligned}
k_{11}(u) & =\frac{1}{2} \mathrm{e}^{-\frac{2}{3} u} \frac{\cosh \eta+\mathrm{e}^{2 u} \cosh 2 \eta}{\cosh \left(u-\frac{1}{2} \eta\right) \cosh \left(u+\frac{3}{2} \eta\right)} \\
k_{33}(u) & =\frac{1}{2} \mathrm{e}^{\frac{2}{3} u} \frac{\cosh \eta+\mathrm{e}^{-2 u} \cosh 2 \eta}{\cosh \left(u-\frac{1}{2} \eta\right) \cosh \left(u+\frac{3}{2} \eta\right)} \\
k_{13}(u) & =\frac{1}{2} \frac{\beta_{13} \cosh \frac{1}{2} \eta \cosh \frac{3}{2} \eta \sinh 2 u}{\cosh \left(u-\frac{1}{2} \eta\right) \cosh \left(u+\frac{3}{2} \eta\right)} \\
k_{31}(u) & =-\frac{1}{2} \frac{\sinh 2 u}{\beta_{13} \cosh \frac{1}{2} \eta \cosh \frac{3}{2} \eta \cosh \left(u-\frac{1}{2} \eta\right) \cosh \left(u+\frac{3}{2} \eta\right)}
\end{aligned}
$$

for the second solution.

As in the IK model the solutions of this case can be obtained from the type-II solution. Substituting

$$
\beta_{11}=\frac{2 \cosh 2 \eta+2 \sinh \eta+\mathrm{e}^{\eta}}{3 \sinh \frac{1}{2} \eta \sinh \frac{3}{2} \eta} \quad \text { and } \quad \beta_{11}=\frac{2 \cosh 2 \eta-2 \sinh \eta-\mathrm{e}^{\eta}}{3 \cosh \frac{1}{2} \eta \cosh \frac{3}{2} \eta}
$$

into $(6.16)$ we will get $(\mathbb{D . 2})$ and (D.3), respectively

Case (ii) $\beta_{13} \neq 0$ and $\beta_{31}=0$ : Here we also have one-parameter solutions

$$
K_{I_{b}}=\left(\begin{array}{ccc}
k_{11} & k_{12} & k_{13} \\
0 & 1 & k_{23} \\
0 & 0 & k_{33}
\end{array}\right) \quad \text { and } \quad K_{I_{c}}=\left(\begin{array}{ccc}
k_{11} & 0 & k_{13} \\
0 & 1 & 0 \\
0 & 0 & k_{33}
\end{array}\right)
$$


with amplitudes gives by

$$
\begin{aligned}
& \beta_{13}=\frac{1}{2} \mathrm{e}^{2 \eta} \beta_{12}^{2} \frac{\sinh \frac{1}{2} \eta \sinh \frac{3}{2} \eta}{\sinh \eta} \\
& k_{12}(u)=-\frac{1}{2} \mathrm{e}^{-\frac{1}{3} u} \beta_{12} \frac{\sinh \frac{3}{2} \eta}{\sinh \left(u-\frac{3}{2} \eta\right)} \sinh 2 u \\
& k_{23}(u)=-\frac{1}{2} \mathrm{e}^{\frac{1}{3} u+2 \eta} \beta_{12} \frac{\sinh \frac{3}{2} \eta}{\sinh \left(u-\frac{3}{2} \eta\right)} \sinh 2 u \\
& k_{13}(u)=-\frac{1}{2} \beta_{13} \frac{\sinh \frac{3}{2} \eta}{\sinh \frac{1}{2} \eta} \frac{\sinh \left(u+\frac{1}{2} \eta\right)}{\sinh \left(u-\frac{3}{2} \eta\right)} \sinh 2 u \\
& k_{11}(u)=-\mathrm{e}^{-\frac{2}{3} u} \frac{\sinh \left(u+\frac{3}{2} \eta\right)}{\sinh \left(u-\frac{3}{2} \eta\right)}, \quad k_{33}(u)=-\mathrm{e}^{\frac{2}{3} u} \frac{\sinh \left(u+\frac{3}{2} \eta\right)}{\sinh \left(u-\frac{3}{2} \eta\right)}
\end{aligned}
$$

for the first solution and

$$
\begin{aligned}
\beta_{13} & =\frac{1}{2} \mathrm{e}^{2 \eta} \beta_{12}^{2} \frac{\cosh \frac{1}{2} \eta \cosh \frac{3}{2} \eta}{\sinh \eta} \\
k_{12}(u) & =\frac{1}{2} \mathrm{e}^{-\frac{1}{3} u} \beta_{12} \frac{\cosh \frac{3}{2} \eta}{\cosh \left(u-\frac{3}{2} \eta\right)} \sinh 2 u \\
k_{23}(u) & =\frac{1}{2} \mathrm{e}^{\frac{1}{3} u+2 \eta} \beta_{12} \frac{\cosh \frac{3}{2} \eta}{\cosh \left(u-\frac{3}{2} \eta\right)} \sinh 2 u \\
k_{13}(u) & =-\frac{1}{2} \beta_{13} \frac{\cosh \frac{3}{2} \eta \cosh \left(u+\frac{1}{2} \eta\right)}{\cosh \frac{1}{2} \eta \cosh \left(u-\frac{3}{2} \eta\right)} \sinh 2 u \\
k_{11}(u) & =\mathrm{e}^{-\frac{2}{3} u} \frac{\cosh \left(u+\frac{3}{2} \eta\right)}{\cosh \left(u-\frac{3}{2} \eta\right)}, \quad k_{33}(u)=\mathrm{e}^{\frac{2}{3} u} \frac{\cosh \left(u+\frac{3}{2} \eta\right)}{\cosh \left(u-\frac{3}{2} \eta\right)}
\end{aligned}
$$

for the second solution.

Case (ii) $\beta_{13}=0$ and $\beta_{31} \neq 0$ : Here we have the $K$-matrices

$$
K_{I_{d}}=\left(\begin{array}{ccc}
k_{11} & 0 & 0 \\
k_{21} & 1 & 0 \\
k_{31} & k_{32} & k_{33}
\end{array}\right) \quad \text { and } \quad K_{I_{e}}=\left(\begin{array}{ccc}
k_{11} & 0 & 0 \\
0 & 1 & 0 \\
k_{31} & 0 & k_{33}
\end{array}\right)
$$

whose matrix elements are obtained from (D.6) and (D.7) by the interchange $k_{i j} \leftrightarrow k_{j i}$ and $\beta_{i j} \leftrightarrow \beta_{j i}$. For both $K_{I_{c}}$ and $K_{I_{e}}$ we have the condition

$$
\sinh 2 \eta=0 \quad \text { and } \quad \sinh \eta \neq 0
$$

Case (iv) $\beta_{13}=0$ and $\beta_{31}=0$ : Here we have two diagonal solutions with no free parameters

$$
K_{I_{f}}=\left(\begin{array}{ccc}
k_{11} & 0 & 0 \\
0 & 1 & 0 \\
0 & 0 & k_{33}
\end{array}\right)
$$


where

$$
k_{11}(u)=-\mathrm{e}^{-\frac{2}{3} u} \frac{\sinh \left(u+\frac{3}{2} \eta\right)}{\sinh \left(u-\frac{3}{2} \eta\right)}, \quad k_{33}(u)=-\mathrm{e}^{\frac{2}{3} u} \frac{\sinh \left(u+\frac{3}{2} \eta\right)}{\sinh \left(u-\frac{3}{2} \eta\right)}
$$

for the first solution and

$$
k_{11}(u)=\mathrm{e}^{-\frac{2}{3} u} \frac{\cosh \left(u+\frac{3}{2} \eta\right)}{\cosh \left(u-\frac{3}{2} \eta\right)}, \quad k_{33}(u)=\mathrm{e}^{\frac{2}{3} u} \frac{\cosh \left(u+\frac{3}{2} \eta\right)}{\cosh \left(u-\frac{3}{2} \eta\right)}
$$

for the second solution.

In addition, we have three reduced solutions from the type-II solution. The two first are one-parameter solutions

$$
K_{I I_{a}}=\left(\begin{array}{ccc}
e^{\frac{4}{3} u} & 0 & \frac{1}{2} \beta_{13} \sinh 2 u \\
0 & 1 & 0 \\
0 & 0 & e^{-\frac{4}{3} u}
\end{array}\right), \quad K_{I I_{b}}=\left(\begin{array}{ccc}
e^{\frac{4}{3} u} & 0 & 0 \\
0 & 1 & 0 \\
\frac{1}{2} \beta_{31} \sinh 2 u & 0 & e^{-\frac{4}{3} u}
\end{array}\right)
$$

and the last is a diagonal solution with no free parameter

$$
K_{I I_{c}}=\left(\begin{array}{ccc}
e^{\frac{4}{3} u} & 0 & 0 \\
0 & 1 & 0 \\
0 & 0 & e^{-\frac{4}{3} u}
\end{array}\right)
$$

They belong to the cases (ii), (iii) and (iv), respectively. 


\section{References}

[1] M. Karowski, H. Thun, T. Troung and P. Weisz, Phys. Lett. B67 (1967) 321.

[2] A. B. Zamolodchikov and Al. B. Zamolodchikov, Ann. Phys. 120 (1979) 253.

[3] R. J. Baxter, Exactly solved models in statistical mechanics (Academic Press 1982).

[4] L. D. Faddeev, Integrable models in (1+1)-dimensional quantum field theory, Proceeding, Les Houches XXXIX, (eds J.-Zuber and R. Stora), North-Holland Publ. 1984, p. 561.

[5] P. P. Kulish and E. K. Sklyanin, Lecture Notes in Physics, vol. 151 (Spring 1982), p. 61.

[6] V. V. Bazhanov, Phys. Lett. 159B (1985) 321; Commun. Math. Phys. 113 (1987) 471.

[7] M. Jimbo, Commun. Math. Phys. 102 (1986) 537.

[8] V. Bazhanov and A. Schadrikov, Theor. Math. Phys. 73 (1987) 402.

[9] I. V. Cherednik, Theor. Math. Phys. 61 (1984) 977.

[10] E. K. Sklyanin, J. Phys A: Math. Gen. 21 (1988) 2375.

[11] F. C. Alcaraz, M. N. Barber, M. T. Batchelor, R. J. Baxter and G. R. W. Quispel, J. Math. Phys. A: Math. Gen. 20 (1987) 6397.

[12] L. Mezincescu and R. I. Nepomechie, J. Phys. A: Math. Gen. 24 (1991) L17.

[13] P.P. Kulish, R. Sasaki and C. Schwiebert, J. Math. Phys. 34 (1993)286.

[14] P.P. Kulish and E. K. Sklyanin, J.Phys.A : Math. Gen. 25 (1992)5963.

[15] L. Hlavary, J. Phys. A: Math. Gen. 27 (1994)5645.

[16] I. V. Cherednik, Int. J. Mod. Phys. A7, Suppl. 1B (1992) 707.

[17] S. Ghoshal and A. B. Zamolodchikov, Int. J. Mod. Phys. A9 (1994)3841

[18] T. Inami and K. Konno, J. Phys. A: Math. Gen. 27 (1994) 211.

[19] A. Fring and R. Köberle, Nucl. Phys. B421 (1994)1592. 
[20] M. T. Batchelor, Reflection Equations and Surface Critical Phenomena, condmat/9608067.

[21] H.-Q. Zhou, X.-Y. Ge, J. Links and M. D. Gould, Graded reflection equation algebra and integrable Kondo impurities in the one-dimensional $t$ - $J$ model, condmat/9809056.

[22] H. J. de Vega and A. González-Ruiz, J. Phys. A: Math. Gen. 26 (1993) L519; J. Phys. A: Math. Gen. 27 (1994) 6129.

[23] L. Mezincescu and R. I. Nepomechie, Quntum Field Theory, Statistic Mechanics, Quantum Group and Topology (eds T. Curtright, L. Mezincescu and R. Nepomechie) World Scientific 1992.

[24] T. Inami, S. Odake and Y.-Z. Zhang, Nucl. Phys. B470 (1996) 419.

[25] Cong-xin Liu, Guo-xing Ju, Shi-kun Wang and Ke Wu, Classification of Solutions to Reflection Equation of Two-Component Systems, hep-th/9808083.

[26] M. T. Batchelor, V. Fridkin, A. Kuniba and Y. K. Zhou, Phys. Lett. B376 (1996) 266.

[27] Changrim Ahn and Choong-Ki You, Complete Nondiagonal Reflection Matrices of RSOS/SOS and Hard Hexagonal Models, hep-th/9710024.

[28] J. Abad amd M. Rios, Diagonal solutions to reflection equations in higher spin models, hep-th/9504069.

[29] J. D. Kim, Boundary K-matrix for the quantum Mikhailov-Shabat model , hepth/9412192.

[30] A. B. Zamolodchikov and V. A. Fateev, Sov. J. Nucl. Phys. 32 (1980) 298.

[31] P. P. Kulish and E. K. Sklyanin, J. Sov. Math. 19 (1982) 1596.

[32] A. G. Izergin and V. E. Korepin, Commun. Math. Phys. 79 (19981) 303.

[33] L. Mezincescu , R. I. Nepomechie, and V. Rittenberg, Phys. Lett. A147 (1990) 70.

[34] L. Mezincescu and R. I. Nepomechie, Int. J. Mod. Phys. A6 (1991)5231. 